\title{
Scattering Theory in the Energy Space for a Class of Non-Linear Wave Equations
}

\author{
J. Ginibre ${ }^{1}$ and G. Velo ${ }^{2}$ \\ ${ }^{1}$ Laboratoire de Physique Théorique et Hautes Energies, ${ }^{\star}$ Université de Paris XI, Bâtiment 211, \\ F-91405 Orsay, France \\ ${ }^{2}$ Dipartimento di Fisica, Università di Bologna and INFN, Sezione di Bologna, Italy
}

\begin{abstract}
We study the asymptotic behaviour in time of the solutions and the theory of scattering in the energy space for the non-linear wave equation

$$
\square \varphi+f(\varphi)=0
$$

in $\mathbb{R}^{n}, n \geqq 3$. We prove the existence of the wave operators, asymptotic completeness for small initial data and, for $n \geqq 4$, asymptotic completeness for arbitrarily large data. The assumptions on $f$ cover the case where $f$ behaves slightly better than a single power $p=1+4 /(n-2)$, both near zero and at infinity (see (1.5), (1.6) and (1.8)).
\end{abstract}

\section{Introduction}

A large amount of work has been devoted to the theory of scattering for the nonlinear wave (NLW) equation (or non-linear massless Klein-Gordon equation)

$$
\square \varphi \equiv \ddot{\varphi}-\Delta \varphi=-f(\varphi),
$$

where $\varphi$ is a complex valued function defined in space time $\mathbb{R}^{n+1}$, the upper dot denotes the time derivative, $\Delta$ is the Laplace operator in $\mathbb{R}^{n}$ and $f$ is a non-linear complex valued function, a typical form of which is

$$
f(\varphi)=\lambda \varphi|\varphi|^{p-1}
$$

with $1 \leqq p<\infty$. We refer to a previous paper [12] for a more detailed introduction and a comprehensive bibliography. It is known $[9,10]$ that the Cauchy problem for the equation (1.1) with initial data $\left(\varphi\left(t_{0}\right), \dot{\varphi}\left(t_{0}\right)\right)=\left(\varphi_{0}, \psi_{0}\right)$ at time $t_{0}$ in the space $H^{1} \oplus L^{2}$ has a unique solution $(\varphi, \dot{\varphi}) \in \mathscr{C}\left(\mathbb{R}, H^{1} \oplus L^{2}\right)$ under assumptions on $f$ which reduce to $\lambda \geqq 0$ and to

$$
0 \leqq p-1<\left\{\begin{array}{lll}
4 /(n-2) & \text { if } & n \geqq 3 \\
\infty & \text { if } & n \leqq 2
\end{array}\right.
$$

\footnotetext{
* Laboratoire associé au Centre National de la Recherche Scientifique
} 
in the special case (1.2). The theory of scattering for the equation (1.1) then gives rise to two main questions. The first one is to prove the existence of dispersive solutions, namely of solutions that behave as solutions of the free wave equation $\square \varphi=0$ at $+\infty$ or $-\infty$ in time, or equivalently to prove the existence of the wave operators. That result is generally proved by solving the Cauchy problem locally at infinity, namely with large (possibly infinite) initial time, by a contraction method in a space of functions exhibiting a suitable time decay, and then by extending those solutions to all times by a standard globalisation method using the available conservation laws, mainly the conservation of the energy. In order to implement the contraction argument at infinity, one has to assume that the interaction $f$ satisfies a suitable condition of decay at infinity in space, which takes the form of a lower bound on $p$ in the special case (1.2). That condition depends on the choice of the space of initial data for the Cauchy problem at infinity, namely of asymptotic states, and is all the more stringent as that space is larger. Using the available estimates for the free wave equation $\square \varphi=0$, it is easy to implement the previous method with a sufficiently small space of initial data under the condition $p>p_{1}(n)$, where $p_{1}(n)$ is the larger root of the equation $[19,20]$

$$
n(n-1) p^{2}-p\left(n^{2}+3 n-2\right)+2=0,
$$

a value which satisfies

$$
1+4 / n<p_{1}(n)<1+4 / n+8 / n^{3} .
$$

The second question which arises in the theory of scattering is to prove asymptotic completeness, namely the fact that all solutions of the equation (1.1) with initial data in a suitable space are actually dispersive at $+\infty$ and $-\infty$. The only treatment of that question available so far is based on the approximate invariance of the equation (1.1) under conformal transformations [24, 15, 12] and requires the use of a natural space $\Sigma$ of initial data (see especially (2.10)-(2.13) in [12]), strictly contained in the space $H^{1} \oplus L^{2}$.

A question left open at this stage is whether a complete theory of scattering for the equation (1.1) can be constructed in a space of initial data as large as the energy space $X_{e}$. That space, which in the present case is slightly larger than $H^{1} \oplus L^{2}$, will be defined in Sect. 2 below (see (2.1)). The solutions of interest, either for the equation (1.1) or for the free equation $\square \varphi=0$, are then the finite energy solutions, which we define as solutions $\varphi$ such that $(\varphi, \dot{\varphi}) \in L_{\text {loc }}^{\infty}\left(I, X_{e}\right)$, where $I$ is the time interval where $\varphi$ is defined. A complete theory of scattering for the equation (1.1) in the energy space has been constructed in [23], but it is restricted to small initial data and to space dimensions $3 \leqq n \leqq 5$. On the other hand, the more difficult part of the problem, namely asymptotic completeness for arbitrarily large initial data in the energy space, has been successfully treated for other non-linear equations, namely the massive non-linear Klein Gordon (NLKG) equation [21, 5, 6, 11], the non-linear Schrödinger (NLS) equation $[8,11,18]$, and the Hartree equation [14]. In all those cases asymptotic completeness is derived through a variant of the original proof of [21] based on the approximate invariance of the various equations under space time dilations.

The purpose of the present paper is to extend that treatment to the NLW 
equation (1.1) and to construct a complete theory of scattering for that equation in the energy space. The proof of existence of dispersive solutions is based on the usual contraction argument and follows $[7,8]$ for the general framework and [23] for some of the estimates. The proof of asymptotic completeness follows the latest version of the method of [21], given in [8] and [11] for the case of the NLS equation and of the NLKG equation respectively. The resulting theory, however, differs from that of the NLKG equation in several respects. The first main difference is that the energy does not contain the $L^{2}$ norm of $\varphi$, and that the latter is in general not bounded in time for finite energy solutions, even for the free equation $\square \varphi=0$. That fact is reflected in the definition (2.1) of the energy space $X_{e}$. Correspondingly, in this paper we never assume the initial data to have finite $L^{2}$ norm. The second difference is that the time decay of the finite energy solutions of the free equation $\square \varphi=0$ is rather weak. As a consequence, the lower bound required on $p$ in order to implement the contraction argument at infinity turns out to be equal to the critical value $p-1=4 /(n-2)$ namely to the upper bound in (1.3). That fact restricts the argument to space dimension $n \geqq 3$. Furthermore, since the extension of the dispersive solutions to all times requires the strict upper bound in (1.3), the whole argument and in particular the construction of the wave operators does not cover the case of a single power interaction like (1.2). The relevant assumptions on $f$ will be stated where needed in Sects. 2-4. They cover for instance the case where

$$
f(\varphi)=\varphi g(|\varphi|)
$$

and $g$ is a smooth non-negative function that behaves as

$$
\begin{cases}g(s)=\lambda_{1} s^{p_{1}-1} & \text { for } \quad 0 \leqq s \leqq a \\ g(s)=\lambda_{2} s^{p_{2}-1} & \text { for } \quad s \geqq 1 / a\end{cases}
$$

for some $a, 0<a<1$, with

$$
0 \leqq p_{2}-1<4 /(n-2)=p_{1}-1 \text {. }
$$

The proof of asymptotic completeness, on the other hand, requires the existence of some norm that decays integrably at infinity in time for solutions of the free equation $\square \varphi=0$. As a consequence, that proof applies only to the case of space dimension $n \geqq 4$. It requires in addition a reinforcement of the assumption on $f$ which takes the form

$$
0 \leqq p_{2}-1<4 /(n-2)<p_{1}-1
$$

in the special case (1.5), (1.6).

This paper is organised as follows. In Sect. 2 we collect and/or derive a number of properties and estimates that are needed in the rest of the paper, in particular space time decay properties of finite energy solutions of the free equation, basic estimates of the non-linear term in (1.1) (Lemma 2.3) and a number of relations between various decay properties of the solutions of the equations (1.1) and $\square \varphi=0$ (Propositions 2.1, 2.2 and 2.3). In Sect. 3 we solve the Cauchy problem for the equation (1.1) with large (possibly infinite) initial time and finite energy initial data. We first solve the local problem at infinity for the critical value of $p$ (Proposition 3.1) and we prove that the solutions thereby obtained are dispersive in a natural sense 
and in particular have asymptotic states in the energy space (Proposition 3.2). As a by-product of those results, we derive global existence and uniqueness of solutions and we prove asymptotic completeness for small finite energy initial data (Proposition 3.3). We then solve the local Cauchy problem at finite times in the subcritical case (Proposition 3.4), we derive the conservation of the energy (Proposition 3.6), and we finally prove the existence of global solutions that are dispersive at $+\infty$ (or $-\infty$ ) and thereby the existence of the wave operators (Proposition 3.7). In Sect. 4 we first derive some uniform boundedness properties of finite energy solutions of the equation (1.1) (Proposition 4.1). We then prove the main result of this paper, namely the fact that for suitable (repulsive) interactions, all finite energy solutions of (1.1) exhibit the same time decay as those of the free equation $\square \varphi=0$ (Proposition 4.2), thereby proving asymptotic completeness. That section follows rather closely Sect. 5 or [8] and Sect. 4 of [11].

We conclude this introduction by giving the main notation used in this paper. For any $r, 1 \leqq r \leqq \infty$, we denote by \|\|$_{r}$ the norm in $L^{r} \equiv L^{r}\left(\mathbb{R}^{n}\right)$. With each $r$ it is convenient to associate the variables $\alpha(r), \beta(r), \gamma(r)$ and $\delta(r)$ defined by

$$
\alpha(r)=2 \beta(r) /(n+1)=\gamma(r) /(n-1)=\delta(r) / n=1 / 2-1 / r .
$$

Of special interest are the values $2^{*}=2 n /(n-2)$ and $r_{s} \equiv 2(n+1) /(n-1)$. We shall use the notation $\gamma_{S} \equiv \gamma\left(r_{S}\right)=(n-1) /(n+1)$ and similarly $\alpha_{S}, \beta_{S}$ and $\delta_{S}$. Pairs of conjugate indices are written as $r$ and $\bar{r}$, where $1 / r+1 / \bar{r}=1$. For any integer $k$, we denote by $H^{k} \equiv H^{k}\left(\mathbb{R}^{n}\right)$ the usual Sobolev spaces. We shall use the homogeneous Besov spaces and the homogeneous Sobolev spaces of arbitrary order and the associated Sobolev inequalities, for which we refer to $[1,3]$ and to the Appendix. We use the notation $\dot{B}_{r}^{\rho} \equiv \dot{B}_{r, 2}^{\rho}\left(\mathbb{R}^{n}\right)$ and $\dot{H}_{r}^{\rho} \equiv \dot{H}_{r}^{\rho}\left(\mathbb{R}^{n}\right)$ for those spaces. For any interval $I \subset \mathbb{R}$, we denote by $\bar{I}$ the closure of $I$ in $\overline{\mathbb{R}}=\mathbb{R} \cup\{+\infty,-\infty\}$. For any interval $I$, for any Banach space $B$, we denote by $\mathscr{C}(I, B)$ the space of continuous functions from $I$ to $B$. For any $q, 1 \leqq q \leqq \infty$ we denote by $L^{q}(I, B)$ (respectively) $\left.L_{\text {loc }}^{q}(I, B)\right)$ the space of measurable functions $\varphi$ from $I$ to $B$ such that $\|\varphi(\cdot) ; B\| \in L^{q}(I)$ (respectively $\left.\|\varphi(\cdot) ; B\| \in L_{\text {loc }}^{q}(I)\right)$. We shall use extensively the following spaces [2]. For any $t \in \mathbb{R}$, let $\gamma_{t}$ be the unit interval with center $t$. For any $m(1 \leqq m \leqq \infty)$ and $q(1 \leqq q \leqq \infty)$, for any Banach space $B$ and for any interval $I \subset \mathbb{R}$, we define $\ell^{m}\left(L^{q}, I, B\right)$ as the space of measurable functions from $I$ to $B$ for which

$$
\left\|\varphi ; \ell^{m}\left(L^{q}, I, B\right)\right\| \equiv \operatorname{Sup}_{s \in \gamma_{0}}\left\{\sum_{z \in \mathbb{Z}}\left(\int_{\left(\gamma_{s+z} \cap I\right)} d t\|\varphi(t) ; B\|^{q}\right)^{m / q}\right\}^{1 / m}
$$

is finite, with obvious modifications if $q$ and/or $m$ is infinite. The spaces $\ell^{m}\left(L^{q}, I, B\right)$ are Banach spaces with the norm defined by (1.9). If $B=\mathbb{C}$, we write $\ell^{m}\left(L^{q}, I\right)$ for $\ell^{m}\left(L^{q}, I, \mathbb{C}\right)$. If $I=\mathbb{R}$, we shall omit $I$ in the notation.

We shall need the operators $\omega=(-\Delta)^{1 / 2}, K(t)=\omega^{-1} \sin \omega t$ and $\dot{K}(t)=\cos \omega t$. The operators $K(t)$ and $\dot{K}(t)$ are bounded and strongly continuous with respect to $t$ in $H^{k}$ for any $k$. Finally, we shall use the notation $\rho_{ \pm}=\operatorname{Max}\{ \pm \rho, 0\}$ for any $\rho \in \mathbb{R}$.

\section{Preliminary Estimates}

In this section we collect a number of definitions and estimates. They concern the finite energy solutions of the free equation $\square \varphi=0$, the finite energy solutions of the 
NLW equation (1.1) and the interaction term $f$ in (1.1). The basic space for the initial data $\left(\varphi_{0}, \psi_{0}\right)=\left(\varphi\left(t_{0}\right), \dot{\varphi}\left(t_{0}\right)\right)$ for the equations $(1.1)$ and $\square \varphi=0$ is the energy space

$$
X_{e}=\left(L^{2 *} \cap \dot{H}_{2}^{1}\right) \oplus L^{2} .
$$

We refer to the Appendix for the meaning of that space as well as for the definition of time derivatives and time integrals. We first consider the equation $\square \varphi=0$.

The solution of the Cauchy problem for $\square \varphi=0$ with initial data $\left(\varphi_{0}, \psi_{0}\right)$ at time zero is given formally by

$$
\varphi^{(0)}(t)=\dot{K}(t) \varphi_{0}+K(t) \psi_{0}
$$

We recall briefly some properties of the operators $K(t), \dot{K}(t)$ and $\exp ( \pm i \omega t)$. The basic estimate for $\exp ( \pm i \omega t)$ is

$$
\left\|\exp ( \pm i \omega t) \varphi ; \dot{B}_{r}^{-\beta(r)}\right\| \leqq C_{r}|t|^{-\gamma(r)}\left\|\varphi ; \dot{B}_{\dot{r}}^{\beta(r)}\right\|
$$

for all $t \in \mathbb{R} \backslash\{0\}, 2 \leqq r \leqq \infty$ and $\varphi \in \dot{B}_{\tilde{r}}^{\beta(r)}$. A proof of (2.3) can be found in [4] and [22]. From this estimate one can derive the following results.

Lemma 2.1. Let $L$ be any of the operators $\omega K(t), \dot{K}(t), \exp ( \pm i \omega t)$. Then, for any $(r, q)$ and $\left(r^{\prime}, q^{\prime}\right)$ with $0 \leqq 2 / q=\gamma(r)<1$ and $0 \leqq 2 / q^{\prime}=\gamma\left(r^{\prime}\right)<1$, for any interval $I \subset \mathbb{R}$, for any $s \in I$, the operator

$$
u \rightarrow \int_{s}^{t} d \tau L(t-\tau) u(\tau)
$$

is bounded from $L^{\bar{q}}\left(I, \dot{B}_{\bar{r}}^{\beta(r)}\right)$ to $L^{q^{\prime}}\left(I, \dot{B}_{r^{\prime}}^{-\beta\left(r^{\prime}\right)}\right)$ with norm uniformly bounded with respect to $I$ and $s$.

Proof. See [13]. See also [17] and [25] for the analogous result for the Schrödinger equation.

Lemma 2.2. (1) Let $r$ and $q$ satisfy $0 \leqq 2 / q=\gamma(r)<1$. Then, for any $\varphi \in L^{2}$, $\exp ( \pm i \omega t) \varphi$ belongs to $L^{q}\left(\mathbb{R}, \dot{B}_{r}^{-\beta(r)}\right)$ and satisfies the estimate

$$
\left\|\exp ( \pm i \omega t) \varphi ; L^{q}\left(\mathbb{R}, \dot{B}_{r}^{-\beta(r)}\right)\right\| \leqq C\|\varphi\|_{2}
$$

(2) Let $r$ and $\rho$ satisfy

$$
\left\{\begin{array}{l}
0 \leqq \delta(r) \leqq n / 2 \\
0 \leqq \rho+\delta(r)-1 \leqq \sigma<1 / 2 \\
\rho \leqq 1-\beta(r)
\end{array}\right.
$$

and let $q$ satisfy $1 / q=\sigma$. Then, for any $\left(\varphi_{0}, \psi_{0}\right) \in X_{e}, \varphi^{(0)}(t) \equiv \dot{K}(t) \varphi_{0}+$ $K(t) \psi_{0} \in L^{q}\left(\mathbb{R}, \dot{B}_{r}^{\rho}\right), \dot{\varphi}^{(0)}(t) \equiv \dot{K}(t) \psi_{0}-\omega^{2} K(t) \varphi_{0} \in L^{q}\left(\mathbb{R}, \dot{B}_{r}^{\rho-1}\right)$ and the following est $i-$ mates hold

$$
\left.\begin{array}{l}
\left\|\varphi^{(0)} ; L^{q}\left(\mathbb{R}, \dot{B}_{r}^{\rho}\right)\right\| \\
\left\|\dot{\varphi}^{(0)} ; L^{q}\left(\mathbb{R}, \dot{B}_{r}^{\rho-1}\right)\right\|
\end{array}\right\} \leqq C\left(\left\|\omega \varphi_{0}\right\|_{2}+\left\|\psi_{0}\right\|_{2}\right) .
$$

Proof. See Lemma 3.1 in [9].

The space-time integrability properties of the solutions of $\square \varphi=0$ are expressed in terms of a family of spaces depending on two parameters, for which one can take any two of the three parameters $r, \rho$ and $\sigma$ connected by (2.5). The allowed region for 


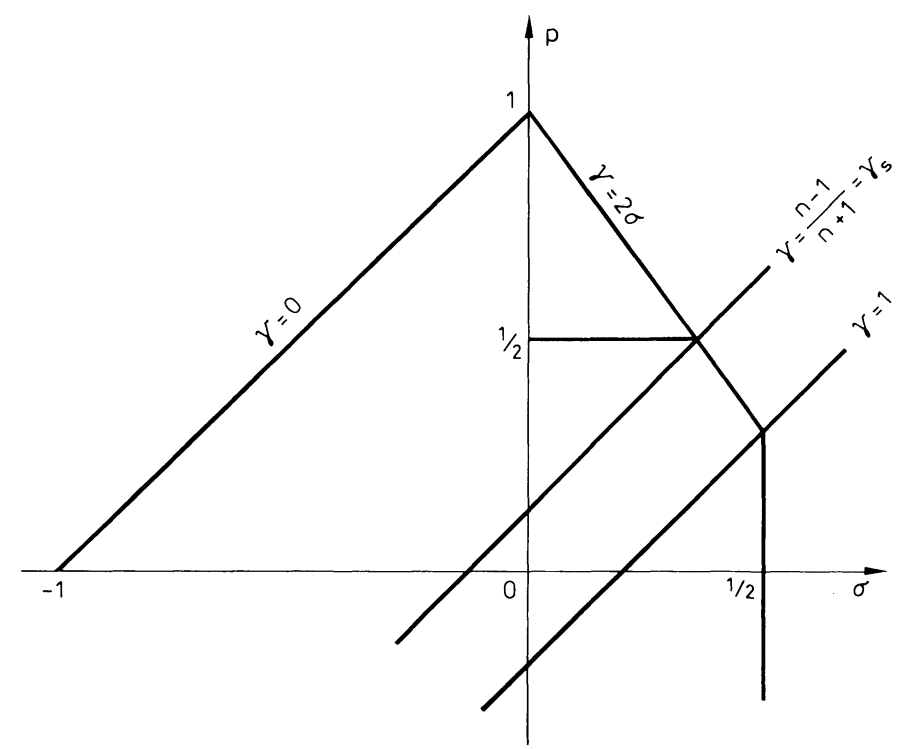

Fig. 1. Space time integrability properties of the solutions of the free wave equation. The case shown is $n=6$

those parameters is shown in Fig. 1 in the $(\rho, \sigma)$ plane. Of special importance is the parameter $\sigma$ which characterizes the regularity in space and in time (in particular the Sobolev inequalities connect spaces with the same value of $\sigma$ ).

We now define the following family of spaces, which embody the dispersive properties of the finite energy solutions of the free equation in the form of the space time integrability properties of Lemma 2.2. For any interval $I \subset \mathbb{R}$, we define

$$
\begin{aligned}
\mathscr{Y}(I)= & \left\{(\varphi, \psi): \varphi \in L^{q}\left(I, \dot{B}_{r}^{\rho}\right) \cap L^{\infty}\left(I, L^{2^{*}}\right) \text { and } \psi \in L^{q}\left(I, \dot{B}_{r}^{\rho-1}\right) \text { for all } r, \rho, \sigma, q\right. \\
& \text { satisfying }(2.5) \text { and } 1 / q=\sigma\} .
\end{aligned}
$$

We also define the corresponding local spaces $\mathscr{Y}_{\text {loc }}(I)$ with $L^{q}$ replaced by $L_{\mathrm{loc}}^{q}$ and $L^{\infty}$ by $L_{\text {loc }}^{\infty}$. Note in particular that $\mathscr{Y}(I) \subset L^{\infty}\left(I, X_{e}\right)$ and $\mathscr{Y}_{\text {loc }}(I) \subset L_{\text {loc }}^{\infty}\left(I, X_{e}\right)$ for all $I \subset \mathbb{R}$. Lemma 2.2 expresses in particular that all solutions of $\square \varphi=0$ with initial data in $X_{e}$ (equivalently all finite energy solutions) belong to $\mathscr{Y}(\mathbb{R})$.

We next show that functions of space-time satisfying some of the properties of Lemma 2.2 possess additional regularity properties corresponding to negative values of $\sigma$. That result will apply to solutions of $\square \varphi=0$ as well as to solutions of (1.1) in suitable circumstances. The next proposition, however, does not make reference to any equation, although the assumptions are inspired by Lemma 2.2.

Proposition 2.1. Let $I$ be an interval of $\mathbb{R}$, possibly unbounded.

(1) Let $k$ satisfy $2(n-1) /(n-2) \leqq k \leqq 2^{*}$, let $\sigma=(n-2) / 2-(n-1) / k$, let $\rho>0$, $\rho^{\prime}>0, \rho+\rho^{\prime} \geqq 1$, let $2 \leqq r, r^{\prime} \leqq \infty$ with $\rho+\delta(r)-1=\rho^{\prime}+\delta\left(r^{\prime}\right)-1=\sigma$ and let $1 / q=\sigma$. Let $\varphi \in L^{q}\left(I, \dot{B}_{r}^{\rho}\right)$ and $\dot{\varphi} \in L^{q}\left(I, \dot{B}_{r^{\prime}}^{\rho^{\prime}-1}\right)$. If $\varphi$, possibly redefined by continuity on a set of measure zero in time, has a representative in $L^{k}$ for some $t_{0} \in I$, then $\varphi$ has a representative in $\mathscr{C}\left(I, L^{k}\right)$ (still denoted by $\left.\varphi\right)$. If $k<2^{*}$, then $\|\varphi(t)\|_{k}^{k}$ is uniformly 
Hölder continuous in $t$ with exponent $k(1-\delta(k))$, and $\varphi$ satisfies the estimate

$$
\|\varphi(t)\|_{k} \leqq C(1+|t|)^{1-\delta(k)}
$$

If $k=2^{*}$ and $I$ is unbounded, say $I=[T, \infty)$, then $\|\varphi(t)\|_{2^{*}}$ has a finite limit as $t \rightarrow \infty$.

(2) Let $k$ satisfy $2 \leqq k \leqq 2(n-1) /(n-2)$. Let $\varphi \in L^{\infty}\left(I, L^{2 *}\right), \dot{\varphi} \in L^{\infty}\left(I, L^{2}\right)$. If $\varphi$, possibly redefined by continuity on a set of measure zero in time, takes its value in $L^{k}$ for some $t_{0} \in I$, then $\varphi \in \mathscr{C}\left(I, L^{k}\right)$. The quantity $\|\varphi(t)\|_{k}^{1 /(1-\delta(k))}$ is uniformly Lipschitz continuous in $t$, and $\varphi$ satisfies the estimate (2.8).

Remark 2.1. The assumptions $\varphi \in L^{q}(I, Y)$ and $\dot{\varphi} \in L^{q}(I, X)$ imply that $\varphi$ can be redefined on a set of measure zero in time so as to belong to $\mathscr{C}(I, X)$. This is the redefinition of $\varphi$ quoted in the proposition.

Proof. (1) We use the regularization operator $R_{j}$ defined in the Appendix. Let $R$ be either $R_{j}$ or $R_{j}-R_{\ell}$ for some $j, \ell$. Then $R \varphi, R \dot{\varphi} \in L^{q}\left(I, H^{m}\right)$ for arbitrary $m$ (we take $m$ sufficiently large) and $R \dot{\varphi}$ is the weak time derivative of $R \varphi$, so that $R \varphi \in \mathscr{C}\left(I, H^{m}\right)$. By an elementary argument of regularization in time we obtain the identity

$$
\|R \varphi(t)\|_{k}^{k}-\|R \varphi(s)\|_{k}^{k}=k \operatorname{Re} \int_{s}^{t} d \tau\left\langle R \dot{\varphi}(\tau), R \varphi(\tau)|R \varphi(\tau)|^{k-2}\right\rangle .
$$

Assuming for definiteness that $s \leqq t$, we estimate

$$
\begin{aligned}
& \left|\|R \varphi(t)\|_{k}^{k}-\|R \varphi(s)\|_{k}^{k}\right| \leqq k \int_{s}^{t} d \tau\left\|R \dot{\varphi}(\tau) ; \dot{B}_{r^{\prime}}^{\rho^{\prime}-1}\right\|\left\|R \varphi(\tau)|R \varphi(\tau)|^{k-2} ; \dot{B}_{r^{\prime}}^{1-\rho^{\prime}}\right\| \\
& \quad \leqq C \int_{s}^{t} d \tau\left\|R \dot{\varphi}(\tau) ; \dot{B}_{r^{\prime}}^{\rho^{\prime}-1}\right\|\left\|R \varphi(\tau) ; \dot{B}_{r}^{\rho}\right\|^{k-1} \\
& \quad \leqq C|t-s|^{1-k \sigma}\left\|R \dot{\varphi} ; L^{q}\left((s, t), \dot{B}_{r^{\prime}}^{\rho^{\prime}-1}\right)\right\|\left\|R \varphi ; L^{q}\left((s, t), \dot{B}_{r}^{\rho}\right)\right\|^{k-1}
\end{aligned}
$$

by duality, by Lemma 3.2 of [9] (an improved version of which is given in Lemma 2.3 Part(1) below) together with Sobolev's inequalities and by the Hölder inequality in time, under the assumptions made on the various indices. In particular the use of Lemma 3.2 of [9] is made possible by the conditions $\rho+\rho^{\prime} \geqq 1, \rho>0$, $\rho^{\prime}>0$ and by the relation between $k$ and $\sigma$, which simply expresses the homogeneity of the estimate. The use of the Hölder inequality is possible under the condition $k \sigma \leqq 1$ which is equivalent to $k \leqq 2^{*}$. The lower limit on $k$ is obtained for $\sigma=0$. Note also that $1-k \sigma=k(1-\delta(k))$.

We first use the estimate (2.10) with $R=R_{j}-R_{\ell}$ and $s=t_{0}$. By the assumption on $\varphi\left(t_{0}\right)$ and the properties of $R_{j}$, the second norm in the first member of (2.10) tends to zero when $j, \ell \rightarrow \infty$. By the assumptions on $\varphi$ and $\dot{\varphi}$, the properties of $R_{j}$ and the Lebesgue dominated convergence theorem, the two norms in the last member of (2.10), for $q<\infty$, or the integral in the last but one member of (2.10), for $q=\infty$, tend to zero when $j, \ell \rightarrow \infty$. Therefore $R_{j} \varphi(t)$ is a Cauchy sequence in $L^{k}$, so that its limit is the canonical representative of $\varphi$. We next use (2.10) with $R=R_{j}-1$. The last member of (2.10) is estimated for any compact subinterval $J \subset \subset I, J$ containing $s=t_{0}$, by

$$
\cdots \leqq C|J|^{1-k \sigma}\left\|R_{j} \dot{\varphi}-\dot{\varphi} ; L^{q}\left(J, \dot{B}_{r^{\prime}}^{\rho^{\prime}-1}\right)\right\|\left\|R_{j} \varphi-\varphi ; L^{q}\left(J, \dot{B}_{r}^{\rho}\right)\right\|^{k-1}
$$


uniformly for $t \in J$ and converges to zero when $j \rightarrow \infty$ by the same argument as before, uniformly for $t \in J$. Therefore $R_{j} \varphi$ converges to $\varphi$ in $L^{k}$ uniformly on the compact subintervals of $I$. Since $R_{j} \varphi \in \mathscr{C}\left(I, L^{k}\right)$ this implies that $\varphi \in \mathscr{C}\left(I, L^{k}\right)$. We finally use (2.10) with $R=R_{j}$ and general $s$ and $t$. Taking the limit $j \rightarrow \infty$ yields the announced Hölder continuity property of $\|\varphi(t)\|_{k}$ and the estimate (2.8) for $k<2^{*}$. If $k=2^{*}$ and $I$ is unbounded, then the limiting estimate shows that $\|\varphi(t)\|_{2^{*}}$ satisfies the Cauchy condition and therefore has a limit as $t \rightarrow \infty$.

(2) In the same way as in Part (1) we obtain (2.9). Assuming for definiteness $s \leqq t$ we estimate

$$
\begin{aligned}
& \left|\|R \varphi(t)\|_{k}^{k}-\|R \varphi(s)\|_{k}^{k}\right| \\
& \quad \leqq k\left\|R \dot{\varphi} ; L^{\infty}\left((s, t), L^{2}\right)\right\|\left\|R \varphi ; L^{\infty}\left((s, t), L^{2 *}\right)\right\|^{v-1} \int_{s}^{t} d \tau\|R \varphi(\tau)\|_{k}^{k-v}
\end{aligned}
$$

by the Hölder inequality, with $v$ given by homogeneity, namely

$$
(v-1)(1 / 2-1 / n)+1-v / k=1 / 2,
$$

or equivalently $v(1-\delta(k))=1$, provided $1 \leqq v \leqq k$, which is equivalent to the assumption made on $k$. By an elementary computation (2.12) implies

$$
\left|\|R \varphi(t)\|_{k}^{v}-\|R \varphi(s)\|_{k}^{v}\right| \leqq v(t-s)\left\|R \dot{\varphi} ; L^{\infty}\left((s, t), L^{2}\right)\right\|\left\|R \varphi ; L^{\infty}\left((s, t), L^{2^{*}}\right)\right\|^{v-1} .
$$

In the same way as in Part (1) we use (2.12) and (2.13) first with $R=R_{j}-R_{\ell}$ and $s=t_{0}$, then with $R=R_{j}-1$ and $s=t_{0}$ and finally with $R=R_{j}$ and general $s$ and $t$, to prove the announced results. QED

In the special case where $k=2^{*}$ and where $I$ is unbounded, one can actually prove under some mild assumptions that $\|\varphi(t)\|_{2^{*}}$ tends to zero when $t$ tends to infinity.

Proposition 2.2. Let $k$ and $s$ satisfy $0 \leqq \delta(k)<1<\delta(s)$ and let $v=(\delta(s)-1)^{-1}+$ $(1-\delta(k))^{-1}$. Let $I$ be an interval of $\mathbb{R}$, possibly unbounded. Let $\varphi \in L_{\text {loc }}^{\infty}\left(I, L^{k}\right) \cap$ $L^{q}\left(I, L^{s}\right)$ with $q(\delta(s)-1)=1$. Assume in addition that

$$
\|\varphi(t)\|_{k} \leqq C_{0}(1+|t|)^{1-\delta(k)}
$$

Then $\varphi$ satisfies the estimate

$$
\int_{I} d t(1+|t|)^{-1}\|\varphi(t)\|_{2^{*}}^{v} \leqq C_{0}^{1 /(1-\delta(k))}\left\|\varphi, L^{q}\left(I, L^{s}\right)\right\|^{q} .
$$

In particular if I is unbounded and if $\|\varphi(t)\|_{2^{*}}$ has a limit as $t$ tends to infinity, that limit is zero.

Proof. For any $a, b \in I, a<b$, we estimate

$$
\int_{a}^{b} d t(1+|t|)^{-1}\|\varphi(t)\|_{2^{*}}^{v} \leqq \int_{a}^{b} d t(1+|t|)^{-1}\|\varphi(t)\|_{k}^{1 /(1-\delta(k))}\|\varphi(t)\|_{s}^{1 /(\delta(s)-1)}
$$

by the Hölder inequality. Then (2.15) follows immediately from (2.16) and (2.14). The last statement of the proposition follows from (2.15). QED

In this paper, we shall use Proposition 2.1 with $k=2 *$ as a preliminary step in the 
proof of energy conservation and with $k=2^{*}-\varepsilon, \varepsilon>0$, so as to be able to apply Proposition 2.2. The result for lower values of $k$, extending down to $k=2$, is given for completeness and would be useful in connection with the Cauchy problem at finite times.

Remark 2.2. By Lemma 2.2, Propositions 2.1 and 2.2 apply in particular to solutions of the free equation $\square \varphi=0$ with initial data in $\left(L^{k} \cap \dot{H}_{2}^{1}\right) \oplus L^{2}$.

In addition to the estimates of Lemma 2.2 on the free equation $\square \varphi=0$, we shall need Besov space estimates for the interaction term of the equation (1.1). Those estimates are given in Lemma 2.3 below which extends Lemma 3.2 of [9]. The statement of that Lemma requires the choice of a representative for the Besov space elements involved. In order to simplify the exposition, that point is not mentioned below. For a treatment thereof, we refer to the Appendix, in particular to Lemma A.3 and to the subsequent discussion.

Lemma 2.3. Let $f \in \mathscr{C}^{1}(\mathbb{C}, \mathbb{C})$ with $f(0)=0$.

(1) Let $g_{1} \in \mathscr{C}\left(\mathbb{C}, \mathbb{R}^{+}\right)$, with $g_{1}(z)=g_{1}(|z|)$ for all $z \in \mathbb{C}, g_{1}$ non-decreasing in $\mathbb{R}^{+}$, and such that

$$
\left|f^{\prime}(z)\right| \equiv \operatorname{Max}\{|\partial f / \partial z|,|\partial f / \partial \bar{z}|\} \leqq g_{1}(z)
$$

for all $z \in \mathbb{C}$. Let $0<\lambda<1$, let $1 \leqq \ell, m, \ell_{i} \leqq \infty(i=1,2)$, with $n / \ell_{1}-\lambda>0$ and $1 / \ell=1 / \ell_{1}+1 / \ell_{2}$. Then

$$
\left\|f(\varphi) ; \dot{B}_{\ell, m}^{\lambda}\right\| \leqq C\left\|\varphi ; \dot{B}_{\ell_{1}, m}^{\lambda}\right\|\left\|g_{1}(\varphi)\right\|_{\ell_{2}}
$$

for all $\varphi$ such that the norms in the right-hand side are finite. The same conclusion holds for $\lambda=1,1<\ell \leqq 2$ and $\ell_{1}=m=2$.

(2) Suppose that there exists $g_{2} \in \mathscr{C}\left(\mathbb{C}, \mathbb{R}^{+}\right)$, with $g_{2}(z)=g_{2}(|z|)$ for all $z \in \mathbb{C}, g_{2}$ nondecreasing in $\mathbb{R}^{+}$, and such that

$$
\left|f^{\prime}\left(z_{1}\right)-f^{\prime}\left(z_{2}\right)\right| \leqq\left|z_{1}-z_{2}\right| \operatorname{Max}\left\{g_{2}\left(z_{1}\right), g_{2}\left(z_{2}\right)\right\}
$$

for all $z_{1}, z_{2} \in \mathbb{C}$. Let $0<\lambda<1$, let $1 \leqq \ell, m, \ell_{i} \leqq \infty(1 \leqq i \leqq 6)$ with $n / \ell_{1}-\lambda>0$ and $1 / \ell=1 / \ell_{1}+1 / \ell_{2}=1 / \ell_{3}+1 / \ell_{5}+1 / \ell_{6}$. Then

$$
\begin{aligned}
\left\|f\left(\varphi_{1}\right)-f\left(\varphi_{2}\right) ; \dot{B}_{\ell, m}^{\lambda}\right\| \leqq & C\left\|\varphi_{1}-\varphi_{2} ; \dot{B}_{\ell_{1}, m}^{\lambda}\right\|\left\{\left\|g_{1}\left(\varphi_{1}\right)\right\|_{\ell_{2}}+\left\|g_{1}\left(\varphi_{2}\right)\right\|_{\ell_{2}}\right\} \\
& +C\left\|\varphi_{1}-\varphi_{2}\right\|_{\ell_{3}} \sum_{i, j=1,2}\left\|\varphi_{i} ; \dot{B}_{\ell_{5}, m}^{\lambda}\right\|\left\|g_{2}\left(\varphi_{j}\right)\right\|_{\ell_{6}},
\end{aligned}
$$

where $g_{1}$ satisfies the assumptions of Part (1), for all $\varphi_{1}, \varphi_{2}$ such that all norms in the right-hand side are finite.

(3) Suppose that for some $v, 0 \leqq v \leqq 1$,

$$
\left|f^{\prime}\left(z_{1}\right)-f^{\prime}\left(z_{2}\right)\right| \leqq C\left|z_{1}-z_{2}\right|^{\nu}
$$

for all $z_{1}, z_{2} \in \mathbb{C}$. Let $0<\lambda<v$, let $1 \leqq \ell, m, \ell_{i} \leqq \infty,(1 \leqq i \leqq 4)$ with $n / \ell_{1}-\lambda>0$, $1 / \ell=1 / \ell_{1}+1 / \ell_{2}=1 / \ell_{3}+1 / \ell_{4}$, and $\ell_{4} v \geqq 1, m v \geqq 1$. Then

$$
\begin{aligned}
\left\|f\left(\varphi_{1}\right)-f\left(\varphi_{2}\right) ; \dot{B}_{\ell, m}^{\lambda}\right\| \leqq & C\left\|\varphi_{1}-\varphi_{2} ; \dot{B}_{\ell_{1}, m}^{\lambda}\right\| \sum_{i}\left\|\varphi_{i}\right\|_{\ell_{2} v}^{v} \\
& +C\left\|\varphi_{1}-\varphi_{2}\right\|_{\ell_{3}} \sum_{i}\left\|\varphi_{i} ; \dot{B}_{\ell_{4} v, m v}^{v / \lambda}\right\|^{v}
\end{aligned}
$$

for all $\varphi_{1}, \varphi_{2}$ such that all norms in the right-hand side are finite. 
Remark 2.3. For the function $g_{1}$ in $\operatorname{Part}(1)$, one can take $g_{1}(z)=\underset{\left|z^{\prime}\right| \leqq|z|}{\operatorname{Sup}}\left|f^{\prime}\left(z^{\prime}\right)\right|$. This function depends only on $|z|$, is non-negative and non-decreasing, and is easily seen to be continuous.

\section{Proof of Lemma 2.3.}

(1) Part (1) is an immediate extension of Lemma 3.1 of [9].

(2) We start from the identity

$$
f\left(\varphi_{1}\right)-f\left(\varphi_{2}\right)=\int_{0}^{1} d \mu\left\{f^{\prime}\left(\mu \varphi_{1}+(1-\mu) \varphi_{2}\right)\right\}\left(\varphi_{1}-\varphi_{2}\right) \equiv \psi\left(\varphi_{1}-\varphi_{2}\right)
$$

and estimate in the same way as in Lemma 3.1 of [9]

$$
\begin{aligned}
\left\|f\left(\varphi_{1}\right)-f\left(\varphi_{2}\right) ; \dot{B}_{\ell, m}^{\lambda}\right\| \leqq & C\left\|\varphi_{1}-\varphi_{2} ; \dot{B}_{\ell_{1}, m}^{\lambda}\right\|\|\psi\|_{\ell_{2}} \\
& +C\left\|\varphi_{1}-\varphi_{2}\right\|_{\ell_{3}}\left\|\psi ; \dot{B}_{\ell_{4}, m}^{\lambda}\right\|
\end{aligned}
$$

with $1 / \ell=1 / \ell_{1}+1 / \ell_{2}=1 / \ell_{3}+1 / \ell_{4}$. We estimate the first norm of $\psi$ in (2.22) by

$$
\begin{aligned}
\|\psi\|_{\ell_{2}} & \leqq\left\|\int d \mu g_{1}\left(\mu \varphi_{1}+(1-\mu) \varphi_{2}\right)\right\|_{\ell_{2}} \\
& \leqq\left\|\operatorname{Max}\left(g_{1}\left(\varphi_{1}\right), g_{1}\left(\varphi_{2}\right)\right)\right\|_{\ell_{2}} \leqq\left\|g_{1}\left(\varphi_{1}\right)\right\|_{\ell_{2}}+\left\|g_{1}\left(\varphi_{2}\right)\right\|_{\ell_{2}} .
\end{aligned}
$$

We then estimate the second norm of $\psi$ in (2.22) by using the integral form of the $\dot{B}$ norm

$$
\left\|\psi ; \dot{B}_{\ell_{4}, m}^{\lambda}\right\| \leqq C\left\{\int_{0}^{\infty} t^{-1} d t\left\{t^{-\lambda} \operatorname{Sup}_{|y| \leqq t}\left\|\psi-\tau_{y} \psi\right\|_{\ell_{4}}\right\}^{m}\right\}^{1 / m},
$$

where $\tau_{y}$ denotes the translation by $y \in \mathbb{R}^{n}$. Now

$$
\begin{aligned}
\left|\psi-\tau_{y} \psi\right| & \leqq \int_{0}^{1} d \mu\left|\mu\left(\varphi_{1}-\tau_{y} \varphi_{1}\right)+(1-\mu)\left(\varphi_{2}-\tau_{y} \varphi_{2}\right)\right| \operatorname{Max}_{i} \operatorname{Max}\left\{g_{2}\left(\varphi_{i}\right), g_{2}\left(\tau_{y} \varphi_{i}\right)\right\} \\
& \leqq \operatorname{Max}_{i, j=1,2}\left|\varphi_{i}-\tau_{y} \varphi_{i}\right| \operatorname{Max}\left\{g_{2}\left(\varphi_{j}\right), g_{2}\left(\tau_{y} \varphi_{j}\right)\right\},
\end{aligned}
$$

so that

$$
\left\|\psi-\tau_{y} \psi\right\|_{\ell_{4}} \leqq 2 \sum_{i, j=1,2}\left\|\varphi_{i}-\tau_{y} \varphi_{i}\right\|_{\ell_{5}}\left\|g_{2}\left(\varphi_{j}\right)\right\|_{\ell_{6}}
$$

with $1 / \ell_{4}=1 / \ell_{5}+1 / \ell_{6}$. Substituting (2.25) into (2.24) yields

$$
\left\|\psi ; \dot{B}_{\ell_{4}, m}^{\lambda}\right\| \leqq C \sum_{i, j=1,2}\left\|\varphi_{i} ; \dot{B}_{\ell_{5}, m}^{\lambda}\right\|\left\|g_{2}\left(\varphi_{j}\right)\right\|_{\ell_{6}} .
$$

The result follows from (2.22), (2.23) and (2.26).

(3) The proof of Part (3) is the same as that of Part (2) except for the treatment of the second norm of $\psi$ in (2.22), which we now estimate differently. From (2.20) we obtain

so that

$$
\left|\psi-\tau_{y} \psi\right| \leqq C \operatorname{Max}\left\{\left|\varphi_{1}-\tau_{y} \varphi_{1}\right|^{v},\left|\varphi_{2}-\tau_{y} \varphi_{2}\right|^{\nu}\right\},
$$

and therefore (cf. (2.24))

$$
\left\|\psi-\tau_{y} \psi\right\|_{\ell_{4}} \leqq C \sum_{i=1,2}\left\|\varphi_{i}-\tau_{y} \varphi_{i}\right\|_{\ell_{4}}^{v}
$$

$$
\left\|\psi ; \dot{B}_{\ell_{4}, m}^{\lambda}\right\| \leqq C \sum_{i}\left\|\varphi_{i} ; \dot{B}_{\ell_{4} v, m v}^{\lambda / v}\right\|^{v}
$$

Substituting (2.23) and (2.27) into (2.22) yields (2.21). QED 
Lemma 2.3 has been tailored to cover the case of the interactions $f$ that we want to consider. In fact we shall assume that $f$ satisfies the following conditions.

(A1) $f \in \mathscr{C}^{1}(\mathbb{C}, \mathbb{C}), f(0)=f^{\prime}(0)=0$ and $f$ satisfies the following estimates

$$
\left|f^{\prime}(z)\right|=\operatorname{Max}\left\{(|(\partial f / \partial z)|,|(\partial f / \partial \bar{z})|\} \leqq C|z|^{p-1}\right.
$$

for some $p \geqq 1$ and for all $z \in \mathbb{C}$.

$$
\left|f^{\prime}\left(z_{1}\right)-f^{\prime}\left(z_{2}\right)\right| \leqq \begin{cases}C\left|z_{1}-z_{2}\right|\left(\left|z_{1}\right|^{p-2}+\left|z_{2}\right|^{p-2}\right) & \text { if } p \geqq 2 \\ C\left|z_{1}-z_{2}\right|^{p-1} & \text { if } p \leqq 2 .\end{cases}
$$

Note that (b) implies (a). We have nevertheless stated (a) and (b) separately because we shall need only (a) in some of the applications.

Remark 2.3'. The assumption (A1) is satisfied in particular by $f(z)=z^{p-q} \bar{z}^{q}$ for fixed $q \in \mathbb{R}$ and $p-2 q \in \mathbb{Z}$. This is obvious for Part (a) and for Part (b) in the case $p \geqq 2$. Part (b) in the case $p<2$ requires a separate argument given in the next lemma.

Lemma 2.4. Let $h(z)=|z|^{v-m} z^{m}$, where $0 \leqq v \leqq 1, m \in \mathbb{Z}$. Then

$$
\left|h\left(z_{1}\right)-h\left(z_{2}\right)\right| \leqq\left\{\begin{array}{lll}
\left|z_{1}-z_{2}\right|^{v} & \text { if } & m=0 \\
2^{1-v}|m|^{v}\left|z_{1}-z_{2}\right|^{v} & \text { if } & m \neq 0 .
\end{array}\right.
$$

Proof. If $m=0,(2.28)$ follows from

$$
\left.|| z_{1}\right|^{v}-\left|z_{2}\right|^{v}|\leqq|\left|z_{1}\right|-\left|z_{2}\right|^{v} \leqq\left|z_{1}-z_{2}\right|^{v} .
$$

Let now $m \neq 0$. By scaling it suffices to consider the case where $z_{1}=z=\rho e^{i \theta}$ and $z_{2}=1$. We first consider the case $m=1$ where it suffices to prove that

or equivalently

$$
\left|\rho^{v} e^{i \theta}-1\right| \leqq 2^{1-v}\left|\rho e^{i \theta}-1\right|^{\nu}
$$

$$
\cosh v \sigma-\cos \theta \leqq 2^{1-v}(\cosh \sigma-\cos \theta)^{v}
$$

with $\rho=e^{\sigma}$. Now the quantity $(\cosh v \sigma-\cos \theta)(\cosh \sigma-\cos \theta)^{-v}$ regarded as a function of $\cos \theta$ is easily seen to take its maximum for $\cos \theta=-1$. In that case (2.29) reduces to

$$
\rho^{v}+1 \leqq 2^{1-v}(\rho+1)^{v}
$$

which follows from the concavity of the function $\rho \rightarrow \rho^{\nu}$. This proves (2.29). The case of general $m \neq 0$ follows from the case $m=1$. In fact (2.29) with $\theta$ replaced by $m \theta$ becomes

$$
\left|\rho^{v} e^{i m \theta}-1\right| \leqq 2^{1-v}\left|\rho e^{i m \theta}-1\right|^{v}
$$

Then

$$
\begin{aligned}
\left|\rho e^{i m \theta}-1\right|^{2} & =(\rho-1)^{2}+4 \rho \sin ^{2}(m \theta / 2) \\
& \leqq m^{2}\left\{(\rho-1)^{2}+4 \rho \sin ^{2}(\theta / 2)\right\}=m^{2}\left|\rho e^{i \theta}-1\right|^{2},
\end{aligned}
$$

since $|m| \geqq 1$ and $|\sin m \theta| \leqq|m \sin \theta|$ for all $\theta \in \mathbb{R}$ and $m \in \mathbb{Z}$. Substituting (2.32) into (2.31) yields (2.28) in the special case considered and therefore in general. QED

We shall study the Cauchy problem for the equation (1.1) in the form of the 
integral equation

$$
\varphi=A\left(t_{0}, \varphi^{(0)} ; \varphi\right)
$$

where $t_{0} \in \mathbb{R}, \varphi^{(0)}$ is a finite energy solution of the free equation $\square \varphi=0$ and

with

$$
A\left(t_{0}, \varphi^{(0)} ; \varphi\right) \equiv \varphi^{(0)}+F\left(t_{0} ; \varphi\right)
$$

$$
F\left(\left(t_{0} ; \varphi\right)(t)=-\int_{t_{0}}^{t} d \tau K(t-\tau) f(\varphi(\tau)) .\right.
$$

Under the assumption (A1a) and for suitable $\varphi$, the integral (2.35) exists in a weak sense and the integral equation (2.33) is essentially equivalent to the differential equation (1.1) supplemented by the initial conditions (see the Appendix).

In order to formulate the theory of scattering and in particular the existence of asymptotic states for the equation (1.1) it is essential to introduce the interaction representation in the first order formalism. For that purpose the generic solution of (2.33) will be represented as a two component vector

$$
\Phi=\left(\begin{array}{c}
\varphi \\
\psi
\end{array}\right)
$$

where $\psi$ will turn out to be $\dot{\varphi}$ as a consequence of the equation. The initial condition for $\Phi$ is

$$
\Phi^{(0)}=\left(\begin{array}{c}
\varphi^{(0)} \\
\dot{\varphi}^{(0)}
\end{array}\right),
$$

the free evolution operatore $U(t)$ is defined by

$$
U(t)=\left(\begin{array}{ll}
\dot{K}(t) & K(t) \\
-\omega^{2} K(t) & \dot{K}(t)
\end{array}\right),
$$

and is a strongly continuous unitary group in $X_{e}$, the interaction term becomes the two component vector

$$
f_{0}(\varphi)=\left(\begin{array}{c}
0 \\
f(\varphi)
\end{array}\right)
$$

The relevant integral equation is

$$
\Phi(t)=\Phi^{(0)}(t)-\int_{t_{0}}^{t} d \tau U(t-\tau) f_{0}(\varphi(\tau)),
$$

the first component of which coincides with the previous one (2.33), while the second component is simply the time derivative of the first one. We introduce the interaction representation by defining

$$
\tilde{\Phi}(t) \equiv U(-t) \Phi(t)=\left(\begin{array}{c}
\tilde{\varphi}(t) \\
\tilde{\psi}(t)
\end{array}\right) .
$$

An elementary computation shows that the differential equation for $\tilde{\Phi}$ is

$$
\dot{\tilde{\Phi}}(t)=U(-t) f_{0}(\varphi(t))
$$


and the corresponding integral equation is

$$
\tilde{\Phi}(t)=\tilde{\Phi}\left(t_{0}\right)-\int_{t_{0}}^{t} d \tau U(-\tau) f_{0}(\varphi(\tau))
$$

The final result of this section expresses the following fact: if a finite energy solution of the integral equation (2.33) satisfies the space-time integrability properties of Lemma 2.2 for one pair of values of $(\rho, \sigma)$ in a suitable restricted set, either locally or globally in time, then it satisfies those properties for all values of $\rho$ and $\sigma$ in the allowed region (2.5), namely it belongs either to $\mathscr{Y}_{\text {loc }}(I)$ or to $\mathscr{Y}(I)$ as defined by (2.7). Furthermore $\widetilde{\Phi}$ has some continuity properties which in some cases imply the existence of asymptotic states.

Proposition 2.3. (1) Let $f$ satisfy (A1a) with

$$
2 /(n-2) \leqq p-1 \equiv 2(2-\zeta) /(n-2) \leqq 4 /(n-2),
$$

or equivalently $0 \leqq \zeta \leqq 1$. Let $\rho, \sigma, r$ and $q$ satisfy $(2.5), 1 / q=\sigma$ and

$$
0 \leqq 1-\zeta-p \sigma=\rho(n-1) /(n+1)<1 / 2 .
$$

Let $I$ be an interval of $\mathbb{R}, t_{0} \in I$, let $\varphi^{(0)}$ be a finite energy solution of $\square \varphi=0$ and let $\varphi \in L_{\mathrm{loc}}^{q}\left(I, \dot{B}_{r}^{\rho}\right)$ be solution of $(2.33)$. Then $\Phi \equiv(\varphi, \dot{\varphi}) \in \mathscr{Y}_{\mathrm{loc}}(I)$. Furthermore possibly after a redefinition of $\Phi$ (and $\widetilde{\Phi})$ by continuity on a set of measure zero in time, $\tilde{\Phi}$ has a representative, still denoted by $\widetilde{\Phi}$, which belongs to $\mathscr{C}^{\zeta}\left(I, X_{e}\right)$ uniformly in compact subintervals of $I$.

(2) Let $f$ satisfy (A1a) with $p-1=4 /(n-2)$. Let $\rho, \sigma, r$ and $q$ satisfy $(2.5), 1 / q=\sigma$ and

$$
0 \leqq 1-\sigma(n+2) /(n-2)=\rho(n-1) /(n+1) .
$$

Let $I$ be an interval of $\mathbb{R}, t_{0} \in \bar{I}$, let $\varphi^{(0)}$ be a finite energy solution of $\square \varphi=0$ and let $\varphi \in L^{q}\left(I, \dot{B}_{r}^{\rho}\right)$ be solution of $(2.33)$. Then $\Phi \equiv(\varphi, \dot{\varphi}) \in \mathscr{Y}(I)$. Furthermore, possibly after a redefinition of $\Phi$ (and $\widetilde{\Phi}$ ) by continuity on a set of measure zero in time, $\widetilde{\Phi}$ has a representative, still denoted by $\widetilde{\Phi}$, which belongs to $\mathscr{C}\left(\bar{I}, X_{e}\right)$. In particular, if $I$ is unbounded, say $I=[T, \infty)$, then $\widetilde{\Phi}(t)$ has a strong limit in $X_{e}$ when $t \rightarrow \infty$.

Proof. The proof of both parts follows from a common set of estimates with $0 \leqq \zeta \leqq 1$ for Part (1) and $\zeta=0$ for Part (2). We first consider the space-time integrability properties of $\varphi$ and $\dot{\varphi}$. It is sufficient to prove those properties in the case $\rho^{\prime}=1-\beta\left(r^{\prime}\right)$. The same properties for general values of $\rho^{\prime}$ and $r^{\prime}$ follow from the latter by the Sobolev inequalities. For that purpose we let $2 \leqq r^{\prime}<\infty, 2 / q^{\prime}=\gamma\left(r^{\prime}\right)$ and

$$
\left\{\begin{array}{l}
0 \leqq \gamma(\ell)<1 \\
2 / m=\gamma(\ell)+2 \zeta
\end{array}\right.
$$

From the integral equation (2.33) and from its analogue for $\dot{\varphi}$ it follows that

$$
\left.\begin{array}{l}
\left\|\varphi ; L^{q^{\prime}}\left(I, \dot{B}_{r^{\prime}}^{1-\beta\left(r^{\prime}\right)}\right)\right\| \\
\left\|\dot{\varphi} ; L^{q^{\prime}}\left(I, \dot{B}_{r^{\prime}}^{-\beta\left(r^{\prime}\right)}\right)\right\|
\end{array}\right\} \leqq C\left\{\left\|\Phi^{(0)}(0) ; X_{e}\right\|+|I|^{\zeta}\left\|f(\varphi) ; L^{\bar{m}}\left(I, \dot{B}_{\bar{\ell}}^{\beta(\ell)}\right)\right\|\right\},
$$

where the first norm on the right-hand side has been estimated by using Lemma 2.2, and the second one by using Lemma 2.1 followed by the Hölder inequality in time. 
Thus we are led to estimate the last norm in (2.44). By Lemma 2.3 Part (1) and by the assumption (A1a) we estimate

$$
\left\|f(\varphi) ; L^{\bar{m}}\left(I, \dot{B}_{\bar{\ell}}^{\beta(\ell)}\right)\right\| \leqq C\left\|\varphi ; L^{q}\left(I, \dot{B}_{r}^{\rho}\right)\right\|^{p}
$$

under the conditions

$$
\left\{\begin{array}{l}
0 \leqq \rho=\beta(\ell)<1 \\
p(n / 2-1-\sigma)=n / \bar{\ell}-\beta(\ell) \\
p \bar{m}=q .
\end{array}\right.
$$

By an elementary computation one can find $m$ and $\ell$ satisfying (2.43) and (2.46) under the assumption (2.42) of the proposition for $0 \leqq \zeta \leqq 1$. More precisely, if $\ell$ and $m$ are defined by the first and last lines of (2.46), then the middle line thereof and the last line of (2.43) both reduce to the equality in (2.42). This proves that $\Phi=(\varphi, \dot{\varphi})$ belongs to $\mathscr{Y}_{\text {loc }}(I)$ under the assumptions of Part (1) and to $\mathscr{Y}(I)$ under the assumptions of Part (2). In particular, $\Phi$ and therefore $\widetilde{\Phi}$ have a representative, still denoted by the same symbol, which belongs to $L_{\text {loc }}^{\infty}\left(I, X_{e}\right)$ or to $L^{\infty}\left(I, X_{e}\right)$. We now turn to the continuity properties of $\widetilde{\Phi}$, which follow from the integral equation (see (2.39))

$$
\tilde{\Phi}(t)-\tilde{\Phi}(s)=-\int_{s}^{t} d \tau U(-\tau) f_{0}(\varphi(\tau))
$$

Applying the regularizing operator $H_{j}$ (see the Appendix) to (2.47) we obtain

$$
H_{j}(\tilde{\Phi}(t)-\tilde{\Phi}(s))=-\int_{s}^{t} d \tau U(-\tau) H_{j} f_{0}(\varphi(\tau)) .
$$

Now $f(\varphi) \in L^{\bar{m}}\left(I, \dot{B}_{\bar{\ell}}^{\beta(\ell)}\right)$ by $(2.45)$, and therefore $H_{j} f(\varphi) \in L^{\bar{m}}\left(I, \dot{H}_{2}^{k}\right)$ for any $k \in \mathbb{Z}$, by the Sobolev inequalities and the definition of $H_{j}$. In particular, the integral in (2.48) is a strong integral in $X_{e}$. Taking the norm in $X_{e}$ of both members of (2.48) we obtain

$$
\left\|H_{j}(\tilde{\Phi}(t)-\tilde{\Phi}(s)) ; X_{e}\right\|^{2}=\int_{s}^{t} d \sigma d \tau\left\langle H_{j} f(\varphi(\sigma)), \dot{K}(\sigma-\tau) H_{j} f(\varphi(\tau))\right\rangle,
$$

after an elementary computation using the definition of $U$ (2.36) and its unitarity in $X_{e}$. We estimate the last integral in (2.49) by (2.3) followed by the HardyLittlewood-Sobolev inequality and the Hölder inequality in time as

$$
\cdots \leqq C|t-s|^{2 \zeta}\left\|H_{j} f(\varphi) ; L^{\bar{m}}\left([s, t] ; \dot{B}_{\bar{\ell}}^{\beta \ell}\right)\right\|^{2}
$$

with the same $m, \ell$ as in (2.44). Taking the limit $j \rightarrow \infty$, using the fact that $H_{j}$ tends to 1 strongly in $\dot{B}_{\bar{\ell}}^{\beta(\ell)}$ and in $X_{e}$ and using the estimate $(2.44)$ on $f(\varphi)$ yields the announced continuity properties of $\tilde{\Phi}$. QED

We next discuss briefly the conditions on $\rho$ and $\sigma$ in Proposition 2.3.

Remark 2.4. For fixed $p$ or equivalently for fixed $\zeta, 0 \leqq \zeta \leqq 1$, the assumptions (2.41), (2.42) constrain $(\rho, \sigma)$ to lie on a line segment in the $(\rho, \sigma)$ plane starting from the point $\{\rho=0, \sigma=((n-2) p-n)) /(2 p)\}$ and ending at the point $\{\rho=(n+1) /(2(n-1))$, $\sigma=((n-2) p-(n+1)) /(2 p)\}$. That segment intersects the region defined by $(2.5)$ in all cases of interest. In particular, for $p=(n+2) /(n-2)$, it intersects the line $\gamma(r)=\gamma_{S}=(n-1) /(n+1)$ within that region at the point $\left\{\rho=n^{2} /\left(2\left(n^{2}+2\right)\right)\right.$, 
$\left.\sigma=(n-2)\left(n^{2}+n+2\right) /\left[2(n+1)\left(n^{2}+2\right)\right]\right\}$. That point is of special interest in connection with the Cauchy problem at infinity.

For the purpose of scattering theory the more relevant part of Proposition 2.3 is Part (2) corresponding to $p-1=4 /(n-2)$. The information relative to $p-1<$ $4 /(n-2)$ namely Part (1) is relevant for the Cauchy problem at finite time. We have not considered the case $p<n /(n-2)$ since the argument would be slightly different (in fact simpler).

\section{The Cauchy Problem at Infinity}

In this section we construct finite energy dispersive solutions of the equation (1.1) by solving the Cauchy problem with large (possibly infinite) initial time and finite energy initial data (or asymptotic states) in the form of the integral equation (2.33). We proceed in two steps. The first step consists in solving the integral equation by a contraction method in a neighborhood of infinity in time ( $+\infty$ for definiteness). For that purpose we define a Banach space of functions exhibiting some of the time decay available from Lemma 2.2 for finite energy solutions of the free equation $\square \varphi=0$ and such that the operator $A\left(t_{0}, \varphi^{(0)} ; \varphi\right)$ defined by $(2.34),(2.35)$ maps a suitable subset of that space into itself and acts there as a contraction. That step can be implemented under the assumption (A1) for the critical value $p-1=4 /(n-2)$ only. Actually the first property has already been obtained to a large extent in Proposition 2.3, which uses basically the estimates of Lemma 2.1. Those estimates however turn out not to be sufficient to yield the contraction property in a reasonable space for high space dimensions (namely $n \geqq 9$ ). We circumvent that difficulty by a direct use of the pointwise estimate (2.3). Since the reproduction property follows from similar, but simpler, estimates than the contraction property, we treat here both properties together, thereby giving a selfcontained treatment not relying on Proposition 2.3. As an immediate by-product of the resolution of the local problem at infinity we obtain global existence and uniqueness of solutions and asymptotic completeness for small initial data in the energy space.

The second step of the argument consists in extending the local solutions previously obtained to all times. This is done by solving the local Cauchy problem at finite times in successive intervals covering the real line, starting from values of $t$ in the interval of existence of the solution one starts from. The possibility of doing so relies on a priori estimates obtained from the conservation of the energy and from estimates of the successive times of local resolution derived therefrom. Unfortunately, no such estimate exists in the critical case $p-1=4 /(n-2)$, thereby precluding the possibility of globalisation in that case. In order to overcome that difficulty, one needs to impose in addition that the assumption (A1) also holds for some subcritical $p$. Accordingly, we solve the local Cauchy problem at finite times under such an assumption. The treatment given in [10] however cannot be applied directly, since we do not assume here the initial data to lie in $L^{2}$, which would be unnatural in the present context. The treatment given in this section is especially tailored for the present purpose, and uses a slight extension of the estimates needed to solve the local problem at infinity in the critical case. In particular, we take initial data in $X_{e}$ only, but we make no effort to cover the case of low values of $p$. 
We first derive an estimate for the integrand in the equation (2.33),

Lemma 3.1. Let $n \geqq 3$. Let $f$ satisfy (A1b) for some $p>1$. Let $\rho, \sigma, r$ satisfy $1+\sigma=$ $\rho+\delta(r)$, and $0<2 \sigma \leqq \gamma(r) \leqq \gamma_{s}$. Define $\rho^{\prime} b y$

$$
(p-1)\left(n / 2-\rho^{\prime}-\delta(r)\right)=1+\gamma(r) .
$$

Then the following estimates hold:

$$
\left\|K(t) f(\varphi) ; \dot{B}_{r}^{\rho}\right\| \leqq C|t|^{-\gamma(r)}\left\|\varphi ; \dot{B}_{r}^{\rho}\right\|\left\|\varphi ; \dot{B}_{r}^{\rho^{\prime}}\right\|^{p-1}
$$

provided $\rho^{\prime} \geqq 0$, for all $\varphi$ such that the right-hand side is finite,

$$
\left\|K(t)\left(f\left(\varphi_{1}\right)-f\left(\varphi_{2}\right)\right) ; \dot{B}_{r}^{\rho}\right\| \leqq C|t|^{-\gamma(r)}\left\|\varphi_{1}-\varphi_{2} ; \dot{B}_{r}^{\rho}\right\| \sum_{i=1,2}\left\|\varphi_{i} ; \dot{B}_{r}^{\rho^{\prime}}\right\|^{p-1}
$$

for $p \geqq 2$, provided $\rho^{\prime} \geqq \sigma+\alpha(r)$, for all $\varphi_{1}, \varphi_{2}$ such that the right-hand side is finite,

$$
\begin{aligned}
& \left\|K(t)\left(f\left(\varphi_{1}\right)-f\left(\varphi_{2}\right)\right) ; \dot{B}_{r}^{\rho}\right\| \\
& \quad \leqq C|t|^{-\gamma(r)}\left\|\varphi_{1}-\varphi_{2} ; \dot{B}_{r}^{\rho}\right\| \sum_{i=1,2} \prod_{ \pm}\left\|\varphi_{i} ; \dot{B}_{r}^{\rho^{\prime} \pm \varepsilon}\right\|^{(p-1) / 2}
\end{aligned}
$$

for $p<2$ and $\varepsilon>0$ sufficiently small, provided $(p-1) \rho^{\prime} \geqq \sigma+\alpha(r)$, for all $\varphi_{1}, \varphi_{2}$ such that the right-hand side is finite.

Remark 3.1. The various restrictions on $\rho^{\prime}$ will be made more explicit in the special cases of interest in Proposition 3.1 and Proposition 3.4.

Proof. We first estimate by (2.3)

$$
\begin{gathered}
\left\|K(t) f(\varphi) ; \dot{B}_{r}^{\rho}\right\| \leqq C|t|^{-\gamma(r)}\left\|f(\varphi) ; \dot{B}_{\bar{r}}^{\lambda}\right\|, \\
\left\|K(t)\left(f\left(\varphi_{1}\right)-f\left(\varphi_{2}\right)\right) ; \dot{B}_{r}^{\rho}\right\| \leqq C|t|^{-\gamma(r)}\left\|f\left(\varphi_{1}\right)-f\left(\varphi_{2}\right) ; \dot{B}_{\bar{r}}^{\lambda}\right\|,
\end{gathered}
$$

where $\lambda \equiv \sigma+\alpha(r)$. Clearly, $0<\lambda<1$. We next estimate the norms in the right-hand sides of (3.5) and (3.6) by Lemma 2.3 with $\ell=\bar{r}$ and $m=2, g_{1}(\varphi)=C|\varphi|^{p-1}$ and $g_{2}(\varphi)=C|\varphi|^{p-2}$ for $p \geqq 2$. In particular we estimate (3.5) by (2.17) and (3.6) by (2.19) or (2.21) depending on whether $p \geqq 2$ or $p<2$. We then estimate the $\dot{B}_{\ell_{1}}^{\lambda}$ norms and the $L^{\ell_{3}}$ norms in terms of the norms in $\dot{B}_{r}^{\rho}$ by using the Sobolev inequalities. Similarly, we estimate all remaining norms in terms of norms in $\dot{B}_{r}^{\rho^{\prime}}$ for suitable $\rho^{\prime}$, thereby continuing (3.5) and (3.6) as

and

$$
\cdots \leqq C|t|^{-\gamma(\boldsymbol{r})}\left\|\varphi ; \dot{B}_{r}^{\rho}\right\|\left\|\varphi ; \dot{B}_{r}^{\rho^{\prime}}\right\|^{p-1}
$$

$$
\cdots \leqq C|t|^{-\gamma(r)}\left\|\varphi_{1}-\varphi_{2} ; \dot{B}_{r}^{\rho}\right\| \sum_{i}\left\|\varphi_{i} ; \dot{B}_{r, m}^{\rho^{\prime}}\right\|^{p-1},
$$

where now $m=\operatorname{Min}\{2,2(p-1)\}$. The conditions for the applicability of the Sobolev inequalities reduce to the homogeneity conditions

$$
\begin{gathered}
n / \ell_{1}-\lambda=n / \ell_{3}=n / r-\rho, \\
n /\left((p-1) \ell_{2}\right)=n / \ell_{5}-\lambda=n /\left((p-2) \ell_{6}\right)=n /\left((p-1) \ell_{4}\right)-\lambda /(p-1)=n / r-\rho^{\prime}
\end{gathered}
$$

and to the conditions $\rho \geqq \lambda, \rho^{\prime} \geqq 0$ for (3.7) and $\rho^{\prime} \geqq \lambda$ or $(p-1) \rho^{\prime} \geqq \lambda$ for (3.8) 
depending on whether $p \geqq 2$ of $p<2$. Under the conditions (3.9) and (3.10), the homogeneity conditions of Lemma 2.3 reduce to

$$
n / r-\rho+(p-1)\left(n / r-\rho^{\prime}\right)=n / \bar{r}-\lambda,
$$

which becomes (3.1) by an elementary computation. The condition $\rho \geqq \lambda$ reduces to $\alpha(r)+\delta(r) \leqq 1$, or equivalently $r \leqq r_{S}$. The conditions on $\rho^{\prime}$ coincide with those stated in the lemma. Finally, in the case when $p<2$, we estimate the last norm in (3.8) as

$$
\left\|\varphi_{i} ; \dot{B}_{r, m}^{\rho^{\prime}}\right\| \leqq \prod_{ \pm}\left\|\varphi_{i} ; \dot{B}_{r}^{\rho^{\prime} \pm \varepsilon}\right\|^{1 / 2}
$$

by Lemma A.1. QED

We now turn to the local Cauchy problem at infinity. Let $0<2 \sigma_{1}<2 \sigma_{2} \leqq$ $\gamma(r)<1$. For any interval $I \subset \mathbb{R}$ we define the space

$$
\mathscr{X}_{0}(I)=\bigcap_{i=1,2} L^{q_{i}}\left(I, \dot{B}_{r}^{\rho_{i}}\right) .
$$

Here and below we consider several values $\rho_{i}, \sigma_{i}, q_{i}$ related by

$$
1 / q_{i}=\sigma_{i}=\rho_{i}+\delta(r)-1
$$

Proposition 3.1. Let $n \geqq 3$. Let $f$ satisfy $(\mathrm{A} 1 \mathrm{~b})$ with $p-1=4 /(n-2)$. Let $r, \sigma_{1}$ and $\sigma_{2}$ satisfy

$$
\begin{gathered}
(n-2) / n \leqq \gamma(r) \leqq \gamma_{s} \\
0<\sigma_{1} \leqq(1-\gamma(r))(n-2) / 4 \leqq \sigma_{2} \leqq \gamma(r) / 2 \quad \text { if } n \leqq 6 \\
0<\sigma_{1} \leqq(1-\delta(r))(n+2) /(n-2)<(1-\gamma(r))(n-2) / 4<\sigma_{2} \leqq \gamma(r) / 2 \quad \text { if } n \geqq 7
\end{gathered}
$$

Let $\varphi^{(0)}$ be a finite energy solution of the free equation $\square \varphi=0$. Then

(1) There exists $T<\infty$ such that, for any $t_{0} \in \bar{I}$, where $I=[T, \infty)$, the equation (2.33) has a unique solution in $\mathscr{X}_{0}(I)$.

(2) For any interval $I$ and for any $t_{0} \in \bar{I}$ the equation (2.33) has at most one solution in $\mathscr{X}_{0}(I)$.

Proof. Part (1) Let $I$ be an interval of $\mathbb{R}$ and let $t_{0} \in \bar{I}$. We first estimate the integral $F\left(t_{0} ; \varphi\right)$ for $\varphi \in \mathscr{X}_{0}(I)$. Let $r$ satisfy (3.13) and let

$$
0<2 \sigma \leqq \gamma(r) \text {. }
$$

It follows from Lemma 3.1 and the Hardy-Littlewood-Sobolev inequality ([16] p. 117) that

$$
\left\|F\left(t_{0} ; \varphi\right) ; L^{q}\left(I, \dot{B}_{r}^{\rho}\right)\right\| \leqq C_{0}(\sigma)\left\|\varphi ; L^{q}\left(I, \dot{B}_{r}^{\rho}\right)\right\|\left\|\varphi ; L^{q^{\prime}}\left(I, \dot{B}_{r}^{\rho^{\prime}}\right)\right\|^{p-1}
$$

with $\rho^{\prime}$ defined by (3.1) and $1 / q^{\prime}=\rho^{\prime}+\delta(r)-1$. The conditions needed to apply that inequality are (1): the homogeneity condition

or equivalently

$$
(p-1) \sigma^{\prime}=1-\gamma(r)
$$

$$
\sigma^{\prime}=(1-\gamma(r))(n-2) / 4
$$


which coincides with (3.1) for the critical value of $p$, and implies $\sigma^{\prime}>0$ and therefore $\rho^{\prime}>0$, and (2): the condition $0<\sigma<1$, which follows from (3.16), and the condition $0<\sigma+(p-1) \sigma^{\prime}<1$, which follows from (3.16) and (3.18). Since $\varphi^{(0)} \in \mathscr{X}_{0}(I)$ by Lemma 2.2, part (2), it follows in particular from (3.16), (3.17) and (3.19) that for $r, \sigma_{1}, \sigma_{2}$ satisfying (3.13) and (3.14), $\mathscr{X}_{0}(I)$ is mapped into itself by the operator $\varphi \rightarrow A\left(t_{0}, \varphi^{(0)} ; \varphi\right)$ defined by $(2.34)$.

We next estimate the difference $F\left(t_{0} ; \varphi_{1}\right)-F\left(t_{0} ; \varphi_{2}\right)$ for $\varphi_{1}, \varphi_{2} \in \mathscr{X}_{0}(I)$. From Lemma 3.1 and the Hardy-Littlewood-Sobolev inequality again it follows that

$$
\begin{aligned}
\left\|F\left(t_{0} ; \varphi_{1}\right)-F\left(t_{0} ; \varphi_{2}\right) ; L^{q}\left(I, \dot{B}_{r}^{\rho}\right)\right\| \leqq & C_{1}(\sigma)\left\|\varphi_{1}-\varphi_{2} ; L^{q}\left(I, \dot{B}_{r}^{\rho}\right)\right\| \\
& \cdot \sum_{i=1,2} \prod_{ \pm}\left\|\varphi_{i} ; L^{q^{\prime}}\left(I, \dot{B}_{r}^{\rho^{\prime} \pm \varepsilon}\right)\right\|(p-1) / 2
\end{aligned}
$$

with $\rho^{\prime}$ again defined by (3.1) or equivalently (3.19), $\varepsilon=0$ for $n \leqq 6, \varepsilon>0$ for $n \geqq 7$,

for $n \leqq 6$ and

$$
\rho^{\prime} \geqq \sigma+\alpha(r)
$$

$$
(p-1) \rho^{\prime} \geqq \sigma+\alpha(r)
$$

for $n \geqq 7$. The conditions needed to apply the Hardy-Littlewood-Sobolev inequality are the same as before. The condition (3.21) reduces to

$$
\sigma \leqq \sigma^{\prime}+1-(n+1) \alpha(r) \equiv \sigma_{3}
$$

and is satisfied in particular for $\sigma=\sigma^{\prime}$, while the condition (3.22) reduces to

$$
\sigma \leqq(1-\delta(r))(n+2) /(n-2) \equiv \sigma_{4}
$$

(cf. the second inequality in (3.15)). An elementary computation shows that $\sigma_{4}<\sigma^{\prime}$ for all $n \geqq 7$ and all $r$ satisfying (3.13).

We can now prove the proposition. We choose $R>0$ sufficiently small so that

$$
\begin{aligned}
& 2 \operatorname{Sup}_{\sigma_{1} \leqq \sigma \leqq \sigma_{2}} C_{0}(\sigma)(2 R)^{p-1} \leqq 1, \\
& 2 \operatorname{Sup}_{\sigma_{1} \leqq \sigma \leqq \sigma_{5}} C_{1}(\sigma)(2 R)^{p-1} \leqq 1,
\end{aligned}
$$

where $\sigma_{5}=\operatorname{Min}\left(\sigma_{2}, \sigma_{3}, \sigma_{4}\right)$. We next choose $T$ sufficiently large so that

$$
\operatorname{Max}_{\sigma=\sigma_{1}, \sigma_{2}}\left\|\varphi^{(0)} ; L^{q}\left(I ; \dot{B}_{r}^{\rho}\right)\right\|=\operatorname{Sup}_{\sigma_{1} \leqq \sigma \leqq \sigma_{2}}\left\|\varphi^{(0)} ; L^{q}\left(I ; \dot{B}_{r}^{\rho}\right)\right\| \leqq R
$$

(the first equality is obtained by interpolation) where $I=[T, \infty)$. It follows now from (3.17) and (3.24) that the operator $\varphi \rightarrow A\left(t_{0}, \varphi^{(0)} ; \varphi\right)$ maps the closed ball $B_{0}(I, 2 R)$ of radius $2 R$ in $\mathscr{X}_{0}(I)$ into itself. Furthermore, in that ball, that operator is a contraction for the norm in the larger space

$$
\mathscr{X}_{1}(I)=L^{q_{1}}\left(I, \dot{B}_{r}^{\rho_{1}}\right) \cap L^{q_{5}}\left(I, \dot{B}_{r}^{\rho_{5}}\right) \text {. }
$$

By standard arguments (cf. Theorem 1 in [17] or Proposition 2.2 in [10]) those two facts imply the existence of a unique solution of the equation (2.33) in $\mathscr{X}_{0}(I)$.

Part (2) follows from Part(1) by standard arguments. QED

Under a mild additional assumption on $r$ and $\sigma_{2}$ the solutions of the 
equation (2.33) in $\mathscr{X}_{0}(I)$ are dispersive in the sense of Proposition 2.3 part (2), and in particular belong to $\mathscr{Y}(I)$ defined by (2.7).

Proposition 3.2. Let $n \geqq 3$. Let $f$ satisfy (A1a) with $p-1=4 /(n-2)$. Let $\mathscr{X}_{0}(\cdot)$ be defined by (3.11) with $r, \sigma_{1}$ and $\sigma_{2}$ satisfying (3.13), (3.14) and (3.15) and in addition the (compatible) condition

$$
(n-2)(2+n \gamma(r)) \leqq 2\left(n^{2}+2\right) \sigma_{2} .
$$

Let $I$ be an interval of $\mathbb{R}, t_{0} \in \bar{I}$, let $\varphi^{(0)}$ be a finite energy solution of $\square \varphi=0$ and let $\varphi \in \mathscr{X}_{0}(I)$ be solution of the equation (2.33). Then $\varphi$ satisfies all the conclusions of Proposition 2.3 part (2).

Proof. In order to be able to apply Proposition 2.3 part (2), it suffices to show that one of the $\dot{B}_{r}^{\rho}$ norms of $\varphi$ available from the definition (3.11), (3.14) (3.15) of $\mathscr{X}_{0}(\cdot)$ controls one of the $\dot{B}_{r}^{\rho}$ norms with $(\rho, \sigma)$ satisfying $(2.42)$ required in the assumptions of that proposition through the Sobolev inequalities, namely has the same $\sigma$ and a larger $\rho$ than the latter. For that purpose, it suffices to show that there exists $\sigma \in\left[\sigma_{1}, \sigma_{2}\right]$ such that

$$
0 \leqq 1-\sigma(n+2) /(n-2) \leqq \rho(n-1) /(n+1)
$$

or equivalently $\sigma \leqq(n-2) /(n+2)$ and

$$
(n-2)(2+n \gamma(r)) \leqq 2\left(n^{2}+2\right) \sigma .
$$

Now it is easily seen that, because of (3.13) and (3.19), $\sigma_{1} \leqq \sigma^{\prime}<(n-2) /(n+2)$, so that it suffices that the conditions (3.29) be satisfied for $\sigma=\sigma_{2}$. That condition reduces to (3.28). The compatibility of (3.14) and (3.15) with (3.28) follows from the inequality

$$
(n-2)(2+n \gamma(r)) \leqq\left(n^{2}+2\right) \gamma(r)
$$

or equivalently $\gamma(r) \geqq(n-2) /(n+1)$ which follows from (3.13). QED

The previous results imply global existence and uniqueness of solutions of the Cauchy problem and asymptotic completeness for small initial data in the energy space.

Proposition 3.3. Let $n \geqq 3$. Let $f$ satisfy $(\mathrm{A} 1 \mathrm{~b})$ with $p-1=4 /(n-2)$. Let $r, \sigma_{1}$ and $\sigma_{2}$ satisfy (3.13), (3.14), (3.15) and (3.28). Then there exists $R_{0}>0$ such that, for any $t_{0} \in \overline{\mathbb{R}}$ and for any $\widetilde{\Phi}_{0}=\left(\tilde{\varphi}_{0}, \widetilde{\psi}_{0}\right) \in X_{e}$ with $\left\|\widetilde{\Phi}_{0} ; X_{e}\right\| \leqq R_{0}$, the equation (2.33) with $\varphi^{(0)}(t)=\dot{K}(t) \tilde{\varphi}_{0}+K(t) \widetilde{\psi}_{0}$ has a unique solution $\varphi$ in $\mathscr{X}_{0}(\mathbb{R})$ (defined by (3.11)). That solution satisfies the conclusions of Proposition 2.3 part (2). In particular the wave operators $\Omega_{ \pm}$(defined as the maps $\tilde{\Phi}_{0} \rightarrow \Phi(0)$ with $t_{0}= \pm \infty$ ) and their inverses $\Omega_{ \pm}^{-1}$ (defined as the maps $\tilde{\Phi}_{0} \rightarrow \widetilde{\Phi}( \pm \infty)$ with $\left.t_{0}=0\right)$ are bijections of $X_{e}$ locally in a neighborhood of zero.

Proof. The result follows immediately from Proposition 2.2 part (2), Proposition 2.3 part (2) and Propositions 3.1 and 3.2. In particular by (2.7), there exists $R_{0}$ such that $\left\|\widetilde{\Phi}_{0} ; X_{e}\right\| \leqq R_{0}$ implies the condition (3.25) needed for Proposition 3.1, with $I=\mathbb{R} . \quad$ QED 
We next turn to the local Cauchy problem at finite times for initial data in $X_{e}$ and subcritical $p$, as explained at the beginning of this section.

Proposition 3.4. Let $n \geqq 3$. Let $f$ satisfy (A1b) with

$$
2 /(n-2)<p-1 \equiv 2(2-\zeta) /(n-2)<4 /(n-2)
$$

or equivalently $0<\zeta<1$. Let $\mathscr{X}_{0}(\cdot)$ be defined by (3.11) with $r, \sigma_{1}, \sigma_{2}$ satisfying

$$
\begin{gathered}
\left\{\begin{array}{l}
2(1-\zeta) \leqq(p+1) \gamma(r) \\
\gamma(r) \leqq \gamma_{s}, \quad \gamma(r)<1-\zeta
\end{array}\right. \\
0<\sigma_{1} \leqq(1-\gamma(r)-\zeta) /(p-1) \leqq \sigma_{2} \leqq \gamma(r) / 2 \quad \text { if } \quad p \geqq 2, \\
\left\{\begin{array}{c}
0<\sigma_{1}<(1-\gamma(r)-\zeta) /(p-1) \leqq \sigma_{2} \leqq \gamma(r) / 2 \\
\sigma_{1} \leqq p(1-\delta(r))-\zeta
\end{array} \quad \text { if } p<2 .\right.
\end{gathered}
$$

Then

(1) For any $R>0$, there exists $T(R)>0$ such that for any $\left(\varphi_{0}, \psi_{0}\right) \in X_{e}$ with $\left\|\left(\varphi_{0}, \psi_{0}\right) ; X_{e}\right\| \leqq R$, for any $t_{0} \in \mathbb{R}$, the equation $(2.33)$ with $\varphi^{(0)}(t)=\dot{K}\left(t-t_{0}\right) \varphi_{0}+$ $K\left(t-t_{0}\right) \psi_{0}$ has a unique solution $\varphi$ in $\mathscr{X}_{0}(I)$, where $I=\left[t_{0}-T(R), t_{0}+T(R)\right]$.

(2) For any interval $I$, any $t_{0} \in I$ and any finite energy solution $\varphi^{(0)}$ of the free equation $\square \varphi=0$, the equation (2.33) has at most one solution in $\mathscr{X}_{0}(I)$.

Proof. Let $I$ be a bounded interval and let $t_{0} \in I$, let $\sigma, r$ satisfy (3.16) and (3.31). Using Lemma 3.1 and the Young inequality, we first estimate for $\varphi \in \mathscr{X}_{0}(I)$,

$$
\left\|F\left(t_{0}, \varphi\right) ; L^{q}\left(I, \dot{B}_{r}^{\rho}\right)\right\| \leqq C_{0}(\sigma)|I|^{\zeta}\left\|\varphi ; L^{q}\left(I, \dot{B}_{r}^{\rho}\right)\right\|\left\|\varphi ; L^{q^{\prime}}\left(I, \dot{B}_{r}^{\rho^{\prime}}\right)\right\|^{p-1}
$$

with $\rho^{\prime}$ defined by $(3.1)$ and $1 / q^{\prime}=\sigma^{\prime}=\rho^{\prime}+\delta(r)-1$, provided

$$
(p-1) \sigma^{\prime}+\zeta=1-\gamma(r)
$$

which coincides with (3.1) under the condition (3.30). The additional conditions required to apply the Young inequality are $0 \leqq \sigma \leqq 1,0<\zeta \leqq 1$ and $0 \leqq \sigma+$ $(p-1) \sigma^{\prime} \leqq 1$ and follow from (3.16), (3.30) and (3.31). Similarly, we estimate for $\varphi_{1}$, $\varphi_{2} \in \mathscr{X}_{0}(I)$,

$$
\begin{aligned}
\left\|F\left(t_{0}, \varphi_{1}\right)-F\left(t_{0}, \varphi_{2}\right) ; L^{q}\left(I, \dot{B}_{r}^{\rho}\right)\right\| \leqq & C_{1}(\sigma)|I|^{\zeta}\left\|\varphi_{1}-\varphi_{2} ; L^{q}\left(I, \dot{B}_{r}^{\rho}\right)\right\| \\
& \cdot \sum_{i=1,2} \prod_{ \pm}\left\|\varphi_{i} ; L^{q^{\prime}}\left(I, \dot{B}_{r}^{\rho^{\prime} \pm \varepsilon}\right)\right\|^{(p-1) / 2}
\end{aligned}
$$

with the same $\rho^{\prime}$ as before, satisfying the condition (3.21) if $p \geqq 2$ and (3.22) if $p<2$, with $\varepsilon=0$ if $p \geqq 2$ and $\varepsilon>0$ if $p<2$, and $1 / q_{ \pm}^{\prime}=\sigma^{\prime} \pm \varepsilon$. The condition (3.21) reduces to (3.23) and is satisfied for $\sigma=\sigma^{\prime}$ as before, while (3.22) now reduces to

$$
\sigma \leqq p(1-\delta(r))-\zeta \equiv \sigma_{4}
$$

We can now prove the proposition. Let $R>0$. Then for any $\left(\varphi_{0}, \psi_{0}\right) \in X_{e}$ with $\left\|\left(\varphi_{0}, \psi_{0}\right) ; X_{e}\right\| \leqq R$, it follows from Lemma $2.2 \operatorname{part}(2)$ that

$$
\sup _{\sigma_{1} \leqq \sigma \leqq \sigma_{2}}\left\|\varphi^{(0)} ; L^{q}\left(\mathbb{R}, \dot{B}_{r}^{\rho}\right)\right\| \leqq c R .
$$


We choose $T \equiv T(R)$ sufficiently small so that

$$
\begin{aligned}
& 2(2 T)^{\zeta} \sup _{\sigma_{1} \leqq \sigma \leqq \sigma_{2}} C_{0}(\sigma)(2 c R)^{p-1} \leqq 1, \\
& 2(2 T)^{\zeta} \sup _{\sigma_{1} \leqq \sigma \leqq \sigma_{5}} C_{1}(\sigma)(2 c R)^{p-1} \leqq 1,
\end{aligned}
$$

where $\sigma_{5}=\operatorname{Min}\left(\sigma_{2}, \sigma_{3}, \sigma_{4}\right)$ as before. It follows from (3.34), (3.36), (3.38), (3.39) and (3.40) that for any $t_{0} \in \mathbb{R}$, and for any $\left(\varphi_{0}, \psi_{0}\right) \in X_{e}$ with $\left\|\left(\varphi_{0}, \psi_{0}\right) ; X_{e}\right\| \leqq R$, the operator $A\left(t_{0}, \varphi^{(0)} ; \varphi\right)$ defined by (2.34) maps the closed ball $B_{0}(I, 2 c R)$ of radius $2 c R$ in $\mathscr{X}_{0}(I)$ into itself, where $I=\left[t_{0}-T(R), t_{0}+T(R)\right]$. Furthermore, in that ball, that operator is a contraction for the norm of the larger space $\mathscr{X}_{1}(I)$ defined by (3.27). The proposition follows from those facts by standard arguments. QED

Remark 3.2. We discuss briefly the conditions (3.31), (3.32) and (3.33), which generalize the simpler conditions (3.13), (3.14) and (3.15) of Proposition 3.1. The condition (3.31), aside from $\gamma(r) \leqq \gamma_{s}$, follows from (3.32) or (3.33) and ensures the existence of $\sigma_{1}$ and $\sigma_{2}$ satisfying (3.32) and (up to a limiting case) the first condition in (3.33). The condition (3.33) implies

$$
p \delta(r)<p-\zeta
$$

which in turn, under the condition (3.31), implies the existence of $\sigma_{1}$ satisfying (3.33). The conditions (3.31) and (3.41) are compatible provided

$$
2 n p(1-\zeta)<(n-1)(p+1)(p-\zeta)
$$

which follows from the fact that $\zeta<1<p$, so that $0<p(1-\zeta)<p-\zeta$, and that $p+1>2(n-1) /(n-2)>2 n /(n-1)$. The condition (3.41) may or may not iollow from (3.31), depending on the values of $p$ and $r$.

Under a mild additional assumption on $r$ and $\sigma_{2}$, the solutions of the equation (2.33) in the previous spaces $\mathscr{X}_{0}(\cdot)$ satisfy the space time integrability properties of Proposition 2.3 part (1). The following proposition is the analogue in the subcritical case of Proposition 3.2.

Proposition 3.5. Let $n \geqq 3$. Let $f$ satisfy (A1a) and (3.30). Let $\mathscr{X}_{0}(\cdot)$ be defined by (3.11) with $r, \sigma_{1}$, and $\sigma_{2}$ satisfying (3.31), (3.32), (3.33) and in addition the (compatible) conditions

$$
\left\{\begin{array}{c}
(2-(n+1) \zeta+n \gamma(r)) \leqq((n+1) p+n-1) \sigma_{2} \\
1 / 2-\zeta<p \sigma_{2} .
\end{array}\right.
$$

Let $I$ be an interval of $\mathbb{R}, t_{0} \in I$, let $\varphi^{(0)}$ be a finite energy solution of $\square \varphi=0$ and let $\varphi \in \mathscr{X}_{0}(I)$ be solution of the equation (2.33). Then $\varphi$ satisfies all the conclusions of Proposition 2.3 part (1).

Proof. In the same way as in the proof of Proposition 3.2, it suffices that there exists $\sigma \in\left[\sigma_{1}, \sigma_{2}\right]$ such that (cf. (2.41))

$$
\begin{aligned}
0 \leqq & 1-\zeta-p \sigma \leqq \rho(n-1) /(n+1), \\
& 1-\zeta-p \sigma<1 / 2 .
\end{aligned}
$$


The second inequality in (3.44) can be rewritten as

$$
(2-(n+1) \zeta+n \gamma(r)) \leqq((n+1) p+n-1) \sigma .
$$

Now it follows from (3.31), (3.32), (3.33) that

$$
\begin{aligned}
p(p-1) \sigma_{1} & \leqq p(1-\zeta-\gamma(r)) \\
& =(p-1)(1-\zeta)+1-\zeta-p \gamma(r) \\
& \leqq(p-1)(1-\zeta-\gamma(r) / 2) \leqq(p-1)(1-\zeta)
\end{aligned}
$$

so that the first inequality in (3.44) is satisfied by $\sigma_{1}$. It suffices therefore that the second inequality in (3.44), or equivalently (3.46), and (3.45) be satisfied by $\sigma_{2}$. Those conditions reduce to (3.42), (3.43) respectively. The compatibility of (3.42) with (3.32), (3.33) is equivalent to the inequality

$$
2(2-(n+1) \zeta) \leqq(n+1)(p-1) \gamma(r)
$$

which is easily seen to follow from (3.31) by an elementary computation. The compatibility of (3.43) with (3.32), (3.33) is equivalent to the inequality

$$
2(1-\zeta)<p \gamma(r)+1,
$$

and follows immediately from (3.31). QED

So far we have studied finite energy solutions of the equation (2.33) without assuming the existence of a conserved energy. However energy conservation is an essential tool for the extension of the local solutions of Proposition 3.1 to all times. We now turn to the proof of that property. For that purpose we need the following assumption on $f$.

(A2) There exists a function $V \in \mathscr{C}^{1}(\mathbb{C}, \mathbb{R})$ such that $V(0)=0, V(z)=V(|z|)$ for all $z \in \mathbb{C}$ and $f(z)=\partial V / \partial \bar{z}$.

We define the energy

$$
E(\varphi, \psi)=\|\psi\|_{2}^{2}+\|\nabla \varphi\|_{2}^{2}+\int d x V(\varphi)
$$

for all $(\varphi, \psi) \in X_{e}$ such that $V(\varphi) \in L^{1}$. We can now prove the conservation of the energy for solutions of (2.33) in the following form.

Proposition 3.6. Let $n \geqq 3$. Let $f$ satisfy (A1b) with $p$ satisfying (2.40) and (A2). Let I be an interval of $\mathbb{R}, t_{0} \in I$, Let $\varphi^{(0)}$ be a finite energy solution of $\square \varphi=0$ with $\varphi^{(0)}\left(t_{0}\right) \in L^{p+1}$ and let $\varphi$ be a solution of the equation (2.33) such that $\Phi=(\varphi, \dot{\varphi}) \in \mathscr{Y}_{\text {loc }}(I)$. Then the following identities hold for all $s$ and $t \in I$,

$$
\begin{gathered}
\int d x V(\varphi(t))-\int d x V(\varphi(s))=2 \operatorname{Re} \int_{s}^{t} d \tau\langle\dot{\varphi}(\tau), f(\varphi(\tau))\rangle, \\
\left\|\Phi(t) ; X_{e}\right\|^{2}-\left\|\Phi(s) ; X_{e}\right\|^{2}=-2 \operatorname{Re} \int_{s}^{t} d \tau\langle\dot{\varphi}(\tau), f(\varphi(\tau))\rangle,
\end{gathered}
$$

where both terms in the left-hand sides of (3.49) and (3.50) are continuous functions of $s$ and $t$ and the integral in the right-hand sides is absolutely convergent. In particular energy conservation holds in the sense that for all $s$ and $t$ in $I$

$$
E(\varphi(t), \dot{\varphi}(t))=E(\varphi(s), \dot{\varphi}(s))
$$


In addition, let $p-1=4 /(n-2)$, let $I$ be unbounded, say $I=[T, \infty)$ and let $\varphi$ be a solution of the equation (2.33) with $\Phi=(\varphi, \dot{\varphi}) \in \mathscr{Y}(I)$. Let however only $t_{0} \in \bar{I}$. Then the same conclusions as above still hold. Furthermore, the kinetic part $\left\|\Phi(t) ; X_{e}\right\|^{2}$ and the potential part $\int d x V(\varphi(t))$ of the energy separately tend to well defined limits when $t \rightarrow \infty$.

Proof. The proof of (3.49) is similar to that of Proposition 2.1 part (1) with $k=p+1$. By the same argument as in the proof of the latter, we obtain

$$
\int d x V\left(R_{j} \varphi(t)\right)-\int d x V\left(R_{j} \varphi(s)\right)=2 \operatorname{Re} \int_{s}^{t} d \tau\left\langle R_{j} \dot{\varphi}(\tau), f\left(R_{j} \varphi(\tau)\right)\right\rangle
$$

where $R_{j}$ is the regularizing operator defined in the Appendix. We next take the limit $j \rightarrow \infty$ in (3.52). By Proposition $2.1 \operatorname{part}(1), \varphi \in \mathscr{C}\left(I, L^{p+1}\right)$ so that $V(\varphi) \in \mathscr{C}\left(I, L^{1}\right)$. Using the identity

$$
V\left(R_{j} \varphi\right)-V(\varphi)=2 \operatorname{Re}\left(R_{j} \bar{\varphi}-\bar{\varphi}\right) \int_{0}^{1} d \mu f\left(\mu R_{j} \varphi+(1-\mu) \varphi\right)
$$

we estimate pointwise in time

$$
\left\|V\left(R_{j} \varphi\right)-V(\varphi)\right\|_{1} \leqq C\left\|R_{j} \varphi-\varphi\right\|_{p+1}\|\varphi\|_{p+1}^{p},
$$

which proves the convergence of the left-hand side of (3.52) to that of (3.49). By the same estimates as in Proposition 2.1 part (1) (see especially (2.10)) and by Lemma 2.3 part (1), the integrand in the right-hand side of (3.52) is bounded uniformly in $j$ and integrably in time according to

$$
\left|\int_{s}^{t} d \tau\left\langle R_{j} \dot{\varphi}(\tau), f\left(R_{j} \varphi(\tau)\right)\right\rangle\right| \leqq C|t-s|^{\zeta}\left\|\dot{\varphi} ; L^{q}\left(I, \dot{B}_{r}^{\rho-1}\right)\right\|\left\|\varphi ; L^{q}\left(I, \dot{B}_{r}^{\rho}\right)\right\|^{p}
$$

with $1 / q=\sigma=(1-\zeta) /(p+1)$ and for instance $\rho=1 / 2$ and $\delta(r)=\sigma+1 / 2$, which satisfies (2.5). Furthermore, for fixed $\tau, R_{j} \dot{\varphi}(\tau)$ tends to $\dot{\varphi}(\tau)$ in $\dot{B}_{r}^{-1 / 2}$ and $f\left(R_{j} \varphi(\tau)\right)$ tends to $f(\varphi(\tau))$ in $\dot{B}_{\bar{r}}^{1 / 2}$ by the properties of $R_{j}$ and Lemma 2.3 part (2), so that the integrand in the left-hand side of (3.52) tends to that in the left-hand side of (3.49). The result then follows from the Lebesgue dominated convergence theorem.

In order to prove (3.50), we start from (2.48) (see also the subsequent comments). By an elementary argument of regularization in time, we obtain

$$
\begin{aligned}
\left\|H_{j} \tilde{\Phi}(t) ; X_{e}\right\|^{2}-\left\|H_{j} \tilde{\Phi}(s) ; X_{e}\right\|^{2} & =2 \operatorname{Re} \int_{s}^{t} d \tau\left\langle H_{j} \dot{\tilde{\Phi}}(\tau), H_{j} \tilde{\Phi}(\tau)\right\rangle \\
& =-2 \operatorname{Re} \int_{s}^{t} d \tau\left\langle H_{j} \Phi(\tau), H_{j} f_{0}(\varphi(\tau))\right\rangle_{e} \\
& =-2 \operatorname{Re} \int_{s}^{t} d \tau\left\langle H_{j} \dot{\varphi}(\tau), H_{j} f(\varphi(\tau))\right\rangle,
\end{aligned}
$$

where $\langle., .\rangle_{e}$ denotes the scalar product in $X_{e}$. We next take the limit $j \rightarrow \infty$ in (3.54). The first member of (3.54) converges to that of (3.50) by the properties of $H_{j}$, the fact that $\tilde{\Phi} \in \mathscr{C}\left(I, X_{e}\right)$ by Proposition 2.3 part (1), and the unitarity of $U(\cdot)$ in $X_{e}$. The last member of (3.54) tends to that of (3.50) by a similar (but simpler) argument as in the proof of (3.49). 
Energy conservation follows by adding (3.49) and (3.50) together and using the definition of the norm in $X_{e}$.

The last statement follows from the fact that $\zeta=0$ and that the integral in the right-hand side of (3.49) and (3.50) is convergent at infinity in the case under consideration. QED

Remark 3.3. In the situation of the second part of the proposition, if $I$ is unbounded, say $I=[T, \infty)$, if $t_{0}$ is finite, and if $\varphi^{(0)}(t) \in L^{k}$ for some $k<2^{*}$ and some $t \in I$, it follows from Propositions 2.1 and 2.2 that $\int d x V(\varphi(t))$ tends to zero when $t$ tends to infinity so that the conservation of the energy takes the familiar form

$$
E(\varphi(t), \dot{\varphi}(t))=\left\|\Phi_{+} ; X_{e}\right\|^{2}
$$

where $\Phi_{+}=\tilde{\Phi}(+\infty)$.

Proposition 3.4 and Remark 3.3 apply in particular to the local dispersive solutions constructed in Proposition 3.1 and to the solutions with small initial data constructed in Proposition 3.3.

We can now prove the existence of global dispersive solutions of the equation (2.33). For simplicity, we restrict our attention to the case of non-negative $V$, the only case anyway that we shall be able to treat in the next section.

Proposition 3.7. Let $n \geqq 3$. Let $f$ satisfy (A1b) both for $p-1=4 /(n-2)$ and for some $p_{2}$ with $p_{2}-1<4 /(n-2)$, and (A2) with $V \geqq 0$. Let $\mathscr{X}_{0}(\cdot)$ be defined by (3.11) with $r, \sigma_{1}, \sigma_{2}$ satisfying (3.13), (3.14), (3.15) and (3.28). Then

(1) For any finite energy solution $\varphi^{(0)}$ of the free equation $\square \varphi=0$, for $t_{0}$ sufficiently large, depending on $\varphi^{(0)}$ and possibly infinite, for any $a \in \mathbb{R}, a \leqq t_{0}$, the equation (2.33) has a unique solution $\varphi$ in $\mathscr{X}_{0}(I)$, with $I=[a, \infty)$. That solution satisfies all the conclusions of Proposition 2.3 part (2), for all such $I$.

(2) The wave operators $\Omega_{ \pm}: \Phi_{ \pm} \equiv \widetilde{\Phi}( \pm \infty) \rightarrow \widetilde{\Phi}(0)$ exist as injective bounded operators in $X_{e}$, and their inverses $\Omega_{ \pm}^{-1}$ are bounded.

Sketch of Proof. Part (1). By Proposition 3.1, there exists $T>0$ depending on $\varphi^{(0)}$ such that all the conclusions of Part (1) hold for $I=[T, \infty)$ and $t_{0} \in I$. In addition the solution $\varphi$ satisfies Proposition 3.4. We then extend those results to arbitrary intervals $[a, \infty)$ by solving the equation (2.33) in successive intervals through the use of Proposition 3.4 applied with $p_{2}-1=2(2-\zeta) /(n-2)$ for $\zeta$ positive and small, starting from $T$. The conservation of the energy (Proposition 3.6) and the condition $V \geqq 0$ ensure that $\left\|\Phi(t) ; X_{e}\right\| \leqq E^{1 / 2}$ uniformly in $t$, where $E$ is the energy of the solution, so that the successive time intervals can be taken of the same length. At each step, the solution comes out to lie in the space $\mathscr{X}_{0}(\cdot)$ used in Proposition 3.4 and satisfies the conclusions of Proposition 2.3 part (2), by Proposition 3.5.

Part (2). The existence and injectivity of the wave operators $\Omega_{ \pm}$follow from Part (1). Boundedness of the $\Omega_{ \pm}$and $\Omega_{ \pm}^{-1}$ follows from energy conservation in the form

$$
E \equiv\left\|\Phi(0) ; X_{e}\right\|^{2}+\int d x V(\varphi(0))=\left\|\Phi_{ \pm}, X_{e}\right\|^{2}+\lim _{t \rightarrow \pm \infty} \int d x V(\varphi(t)),
$$

where the last limit exists by Proposition 3.6. In addition

$$
0 \leqq \int d x V(\varphi(0)) \leqq C\|\varphi(0)\|_{2^{*}}^{2^{*}} \leqq C\left\|\Phi(0) ; X_{e}\right\|^{2^{*}}
$$


so that $\left\|\Phi_{ \pm} ; X_{e}\right\|$ is estimated in terms of $\left\|\Phi(0) ; X_{e}\right\|$, while

$$
\lim _{t \rightarrow \pm \infty} \int d x V(\varphi(t)) \leqq C \lim _{t \rightarrow \pm \infty}\left\|\Phi(t) ; X_{e}\right\|^{2^{*}}=C\left\|\Phi_{ \pm} ; X_{e}\right\|^{2^{*}}
$$

by the continuity of $\widetilde{\Phi}$ from $\bar{I}$ to $X_{e}$, so that conversely $\left\|\Phi(0) ; X_{e}\right\|$ is estimated in terms of $\left\|\Phi_{ \pm} ; X_{e}\right\|$. QED

\section{Time Decay of Solutions and Asymptotic Completeness}

In this section we study the asymptotic behaviour in time of finite energy solutions of the equation (1.1), defined as solutions $\varphi$ of (1.1) such that $\Phi=(\varphi, \dot{\varphi}) \in L^{\infty}\left(\mathbb{R}, X_{e}\right)$. It follows from Propositions 3.4, 3.5 and 3.6 and standard globalisation arguments that for $f$ satisfying the assumptions of Proposition 3.7 and for any $\Phi_{0}=$ $\left(\varphi_{0}, \psi_{0}\right) \in X_{e}$, the equation (1.1) with initial data $\Phi\left(t_{0}\right)=\Phi_{0}$ at some finite initial time $t_{0} \in \mathbb{R}$ has a global solution which is unique in $\mathscr{Y}_{\text {loc }}(\mathbb{R})$ and which is a finite energy solution. We shall see in Proposition 4.1 below that in fact any finite energy solution belongs to $\mathscr{Y}_{\text {loc }}(\mathbb{R})$ and therefore can be recovered by solving the Cauchy problem with finite initial time in the way described above. We first prove that all finite energy solutions of the equation (1.1) satisfy some uniform boundedness properties in the sense that they belong to $\ell^{\infty}\left(L^{q}\left(\dot{B}_{r}^{\rho}\right)\right)$ for suitable values of $r, \rho, q$ and suitable assumptions on the interaction $f$. The method of proof is a direct estimation and does not require the elaborate machinery of $[8,11,21]$. We next prove the main result of this paper, namely the fact that for a class of repulsive interactions, all finite energy solutions of the equation (1.1) are dispersive in the sense that they satisfy the space-time integrability properties previously found for the solutions of the free equation $\square \varphi=0$ (see Lemma 2.2). In the framework of Scattering theory, those properties imply asymptotic completeness in the energy space. The proof relies on the Morawetz-Strauss estimate [21] which is directly related to the approximate dilation invariance of the equation (see Lemma 4.3) and on the finiteness of the propagation speed for the equation (1.1) (see Lemma 4.2). Combining those two estimates one proves that suitable Besov norms of finite energy solutions of the equation (1.1) are arbitrarily small in arbitrarily large time intervals (see Lemma 4.5). That property is exploited through the integral equation (2.33) and for that purpose one needs some additional estimates on the integrand in that equation (see Lemma 4.7). With those estimates available the proof follows step by step the corresponding proof for the NLS equation, given in [8] and for the NLKG equation, given in [11]. The final results are collected in Proposition 4.2.

In this section we shall make repeated use of an additional estimate on the interaction term, which can be stated as Lemma 4.1 below. As a first approximation, the reader can take $\eta=0$ in that Lemma. The case $\eta \neq 0$ is needed only in the proof of Lemma 4.5 below.

Lemma 4.1. Let $n \geqq 3$. Let $f$ satisfy (A1a). Let $0<\lambda \leqq 1, \rho \leqq \lambda, \rho<1,1<\bar{\ell} \leqq 2 \leqq r$, $v<\infty, 0 \leqq \eta \leqq \operatorname{Min}\{\delta(\ell) / n,(p-1) / v\}$ and $0 \leqq \sigma \equiv \rho+\delta(r)-1<1$. Then, for all $\varphi \in \dot{H}_{2}^{1} \cap \dot{\dot{B}_{r}^{\rho}}$, with $\varphi \in L^{v}$ if $\eta>0$, the following inequality holds:

$$
\left\|f(\varphi) ; \dot{B}_{\bar{\ell}}^{\lambda}\right\| \leqq C\left\|\varphi ; \dot{H}_{2}^{1}\right\|^{p-v-\eta v}\left\|\varphi ; \dot{B}_{r}^{\rho}\right\|^{v}\|\varphi\|_{v}^{\eta v}
$$


provided

$(p-1)\left(n / 2-1-\sigma /\left(1+\rho_{-}\right)\right) \leqq \delta(\ell)+\delta(r)(1-\lambda) /(1-\rho)+\eta v\left(\delta(v)-1-\sigma /\left(1+\rho_{-}\right)\right)$

and provided $v \geqq 0$ satisfies

$$
(p-1)(n / 2-1)=1+\delta(\ell)-\lambda+v \sigma+\eta v(\delta(v)-1) .
$$

Proof. We estimate the left-hand side of (4.1) by Lemma 2.3 part (1) as

with

$$
\left\|f(\varphi) ; \dot{B}_{\bar{l}}^{\lambda}\right\| \leqq C\left\|\varphi ; \dot{B}_{k}^{\lambda}\right\|\left\||\varphi|^{p-1}\right\|_{s}
$$

$$
n / s=\delta(\ell)+\delta(k)
$$

and we estimate the last norm in (4.4) by the Hölder inequality as

with

$$
\left\||\varphi|^{p-1}\right\|_{s} \leqq\left\||\varphi|^{p-1-\eta v}\right\|_{u}\|\varphi\|_{v}^{n v}
$$

$$
1 / s-1 / u=\eta
$$

Since $0 \leqq \eta \leqq \delta(\ell) / n$, the conditions (4.5) and (4.7) determine $u$ with $1 \leqq u \leqq \infty$ for any $k \geqq 2$ and $v \geqq 2$. We next estimate the norms of $\varphi$ in $\dot{B}_{k}^{\lambda}$ and $L^{(p-1-\eta v) u}$ by interpolation between the norms of $\varphi$ in $\dot{H}_{2}^{1}$ and in $\dot{B}_{r}^{\rho}$ and by using the Sobolev inequalities if necessary. The interpolation is possible provided

$$
1-\lambda \leqq \delta(k) \leqq \delta(r)(1-\lambda) /(1-\rho)
$$

and

$$
1 \leqq \delta((p-1-\eta v) u) \leqq 1+\operatorname{Min}\left\{\sigma,(\sigma /(1-\rho)\} \equiv 1+\sigma /\left(1+\rho_{-}\right)\right.
$$

or equivalently, after elimination of $u$ through (4.5) and (4.7)

$$
\begin{aligned}
(p-1-\eta v)\left(n / 2-1-\sigma /\left(1+\rho_{-}\right)\right) & \leqq \delta(k)+\delta(\ell)-\eta n \\
& \leqq(p-1-\eta v)(n / 2-1) .
\end{aligned}
$$

The conditions (4.8) and (4.10) constrain $\delta(k)$ to lie in the intersection of two intervals, both of which are non-empty under the assumptions made (in particular $p-1-\eta v \geqq 0$ ). The interpolation is possible, namely the conditions (4.8) and (4.10) are compatible for $k$, under the conditions (4.2) and

$$
(p-1)(n / 2-1) \geqq 1+\delta(\ell)-\lambda+\eta v(\delta(v)-1) .
$$

When possible, the interpolation yields (4.1), where $v$ is defined by the homogeneity condition

$$
n / \bar{\ell}-\lambda=(p-v-\eta v)(n / 2-1)+v(n / r-\rho)+\eta n
$$

or equivalently (4.3), and satisfies $0 \leqq v \leqq p-\eta v$. Finally, the lower interpolation condition (4.11) reduces to the condition $v \geqq 0$. QED

We are now in a position to prove the basic uniform boundedness result for the solutions of the equation (1.1).

Proposition 4.1. Let $n \geqq 3$. Let $f$ satisfy (A1a) with $p$ satisfying (3.30). Let $\varphi$ be a 
solution of the equation (1.1) such that $\Phi \equiv(\varphi, \dot{\varphi}) \in L_{\mathrm{loc}}^{\infty}\left(\mathbb{R}, X_{e}\right)$. Then

(1) $\Phi \equiv(\varphi, \dot{\varphi}) \in \mathscr{Y}_{\text {loc }}(\mathbb{R})$.

Let in addition $n \geqq 4$, let

$$
3 /(n-2)<p-1<4 /(n-2)
$$

or equivalently (see (2.40)) $0<\zeta<1 / 2$, and let $\varphi$ be a finite energy solution. Then (2) For any $r, \rho, \sigma, q$ satisfying (2.5) and $1 / q=\sigma<\gamma(r) / 2, \varphi \in \ell^{\infty}\left(L^{q}\left(\dot{B}_{r}^{\rho}\right)\right)$ and $\dot{\varphi} \in \ell^{\infty}\left(L^{q}\left(\dot{B}_{r}^{\rho-1}\right)\right)$.

Proof. We concentrate on the properties of $\varphi$. Those of $\dot{\varphi}$ are derived in a similar way.

Part (1). The proof is similar to that of Lemma 3.3 of [9]. The additional lower bound on $p$ as compared with that lemma comes from the fact that the $L^{2}$ norm of $\varphi$ is not available in the present case. By interpolation with $\dot{H}_{2}^{1}$ and by the Sobolev inequalities, it suffices to prove the result for $\gamma(r)=1-\varepsilon$ and $0<2 \sigma \leqq \gamma(r)$, with $\varepsilon>0$ and small. For such a value of $r$, we prove it in each bounded interval $I$ for values of $\sigma$ increasing from 0 to $\gamma(r) / 2$ by successive steps of length $\varepsilon$. At each step we estimate the norm of $\varphi$ in $L^{q^{\prime \prime}}\left(I, \dot{B}_{r}^{\rho^{\prime \prime}}\right)$ with $1 / q^{\prime \prime}=\sigma^{\prime \prime}=\sigma^{\prime}+\varepsilon=1 / q^{\prime}+\varepsilon$ by the use of the integral equation (2.33). We estimate the integrand by (2.3) and Lemma 4.1 with $\eta=0$ as

$$
\begin{aligned}
\left\|K(t-\tau) f(\varphi) ; \dot{B}_{r}^{\rho^{\prime \prime}}\right\| & \leqq C|t-\tau|^{-\gamma(\ell)}\left\|f(\varphi) ; \dot{B}_{\frac{\ell}{\ell}}^{\lambda}\right\| \\
& \leqq C|t-\tau|^{-\gamma(\ell)}\left\|\varphi ; \dot{H}_{2}^{1}\right\|^{p-v}\left\|\varphi ; \dot{B}_{r}^{\rho^{\prime}}\right\|^{v}
\end{aligned}
$$

with $\ell \leqq r$ and $\lambda=\sigma^{\prime \prime}+\alpha(\ell)$ so that (4.3) becomes

$$
(p-1)(n / 2-1)=1+\gamma(\ell)+v \sigma^{\prime}-\sigma^{\prime \prime} .
$$

We then estimate

$$
\left\|F\left(t_{0}, \varphi\right) ; L^{q^{\prime \prime}}\left(I, \dot{B}_{r}^{\rho^{\prime \prime}}\right)\right\| \leqq C|I|^{\zeta}\left\|\varphi ; L^{\infty}\left(\mathbb{R}, \dot{H}_{2}^{1}\right)\right\|^{p-v}\left\|\varphi ; L^{q^{\prime}}\left(I, \dot{B}_{r}^{\rho^{\prime}}\right)\right\|^{v}
$$

by the Young inequality, provided $0<\zeta \leqq 1,0 \leqq \sigma^{\prime \prime}, v \sigma^{\prime} \leqq 1$, and

$$
\zeta=1-\gamma(\ell)-v \sigma^{\prime}+\sigma^{\prime \prime},
$$

which coincides with (4.15). One can take for instance $\gamma(\ell)=1-\zeta+\varepsilon$ with $\varepsilon \leqq \zeta / 2$, and $v=1$. The upper interpolation condition (4.2) needed to apply Lemma 4.1 has been shown to hold for that choice and the relevant values of $\sigma^{\prime}, \sigma^{\prime \prime}$ in the proof of Lemma 3.3 of [9]. Substituting the estimate (4.16) into the integral equation (2.33), one obtains for the previous choice $v=1$,

$$
\begin{aligned}
\left\|\varphi ; L^{q^{\prime \prime}}\left(I, \dot{B}_{r}^{\rho^{\prime \prime}}\right)\right\| \leqq & \left\|\varphi^{(0)} ; L^{q^{\prime \prime}}\left(I, \dot{B}_{r}^{\rho^{\prime \prime}}\right)\right\|+C|I|^{\zeta}\left\|\varphi ; L^{\infty}\left(\mathbb{R} ; \dot{H}_{2}^{1}\right)\right\|^{p-1} \\
& \cdot\left\|\varphi ; L^{q^{\prime}}\left(I, \dot{B}_{r}^{\rho^{\prime}}\right)\right\|
\end{aligned}
$$

from which the result follows in a finite number of steps.

Part (2). The proof is similar to that of Proposition 3.1 of [11]. By interpolation and the Sobolev inequalities, it suffices to prove the result for $\gamma(r)=1+\varepsilon$ and $\sigma=1 / 2-\varepsilon$ with $\varepsilon>0$ and small. For such a choice of $r$ and $\sigma$, we estimate $\varphi$ again by the use of the integral equation (2.33). We estimate the integrand by (2.3) and Lemma 4.1 with 
$\eta=0$ as

$$
\left\|K(t-\tau) f(\varphi) ; \dot{B}_{r}^{\rho}\right\| \leqq C|t-\tau|^{-\gamma(t)}\left\|\varphi ; \dot{H}_{2}^{1}\right\|^{p-v}\left\|\varphi ; \dot{B}_{r}^{\rho}\right\|^{v}
$$

with $\ell \leqq r$ and $\lambda=\sigma+\alpha(\ell)$ so that (4.3) becomes

or equivalently

$$
(p-1)(n / 2-1)=1+\gamma(\ell)+(v-1) \sigma,
$$

$$
\zeta=1-\gamma(\ell)-(v-1) \sigma .
$$

We use the estimate (4.19) with different values of $\ell$ and $v$ according to whether $|t-\tau| \leqq 1$ or $|t-\tau| \geqq 1$, with

$$
0 \leqq \nu \leqq 1-\varepsilon / \sigma<1
$$

in both cases. For $|t-\tau| \leqq 1$, we take $0 \leqq \gamma(\ell) \equiv \gamma_{-} \leqq 1-\varepsilon$, which is compatible with (4.22) provided $2 \varepsilon \leqq \zeta \leqq 1+\sigma=3 / 2-\varepsilon$, and therefore under (3.30) for $\varepsilon$ sufficiently small. For $|t-\tau| \geqq 1$, we take $\gamma(\ell) \equiv \gamma_{+}=\gamma(r)=1+\varepsilon$, which is compatible with (4.22) provided $0 \leqq \zeta \leqq \sigma-\varepsilon=1 / 2-2 \varepsilon$, and therefore under (4.13) for $\varepsilon$ sufficiently small. We denote by $v_{ \pm}$the values of $v$ corresponding to the two cases. We now define

$$
\begin{aligned}
k_{0}(t) & \equiv\left\|\varphi^{(0)}(t) ; \dot{B}_{r}^{\rho}\right\|, \\
k(t) & \equiv\left\|\varphi(t) ; \dot{B}_{r}^{\rho}\right\| .
\end{aligned}
$$

We restrict our attention to positive times. Taking the norm in $\dot{B}_{r}^{\rho}$ of both members of the integral equation (2.33) and estimating the integrand by (4.19) we see that for $t \geqq 0, k(t)$ satisfies the integral inequality

$$
k \leqq k_{0}+\mu * \sum_{ \pm} k^{v_{ \pm}}
$$

with

$$
\begin{cases}\mu(t)=C \underset{ \pm}{\operatorname{Min}}|t|^{-\gamma_{ \pm}} \sum_{ \pm}\left\|\varphi ; L^{\infty}\left(\mathbb{R}, \dot{H}_{2}^{1}\right)\right\|^{p-v_{ \pm}} & \text {for } t>0, \\ \mu(t)=0 & \text { for } t \leqq 0 .\end{cases}
$$

In particular, $\mu \in L^{1}\left(\mathbb{R}^{+}\right)$. We now take $a>0$, multiply both members of (4.25) by the characteristic function $\chi_{a}$ of the interval $[0, a]$, and take the norm in $\ell^{\infty}\left(L^{q}\right)$. Applying the Young inequality in the spaces $\ell^{*}\left(L^{*}\right)$ (see for instance Lemma 5.6 of [8]) and the inclusion $\ell^{\infty}\left(L^{q}\right) \subset \ell^{\infty}\left(L^{v q}\right)$ for $v \leqq 1$, we obtain

$$
\left\|\chi_{a} k ; \ell^{\infty}\left(L^{q}\right)\right\| \leqq\left\|k_{0} ; \ell^{\infty}\left(L^{q}\right)\right\|+C \operatorname{Max}_{ \pm}\left\|\varphi ; L^{\infty}\left(\mathbb{R}, \dot{H}_{2}^{1}\right)\right\|^{p-v_{ \pm}}\left\|\chi_{a} k ; \ell^{\infty}\left(L^{q}\right)\right\|^{v_{ \pm}} .
$$

The contribution of the free term is controlled by Lemma 2.2. Since $v_{ \pm}<1$, the lefthand side is bounded uniformly with respect to $a$. This completes the proof. QED

We now prove the finiteness of the propagation speed for the equation (1.1) in the form of local energy conservation. The result given in Lemma 4.2 below, and its formal algebraic derivation are the same as that for the NLKG equation given in Lemma 4.2 of [11]. The actual proof however, is somewhat different because we use a different (more economical) regularization procedure. For any open ball 
$\Omega=B(x, L)$ of center $x$ and radius $L$ in $\mathbb{R}^{n}$, for any $t \in \mathbb{R}$, we define $\Omega_{ \pm}(t)=$ $B(x, L \pm|t|)$ with the convention that $B(x, L)$ is empty if $L \leqq 0$. For any measurable set $\Omega \subset \mathbb{R}^{n}$, for any $(\varphi, \psi) \in X_{e}$, we define

$$
E(\varphi, \psi ; \Omega)=\int_{\Omega} d x\left(|\psi|^{2}+|\nabla \varphi|^{2}+V(\varphi)\right) .
$$

Lemma 4.2. Let $n \geqq 3$. Let $f$ satisfy (A1b) with $p$ satisfying (3.30) and (A2) with $V \geqq 0$. Let $\varphi$ be a finite energy solution of the equation (1.1). Then for any open ball $\Omega \subset \mathbb{R}^{n}$, for any $t \in \mathbb{R}$, the following inequalities hold:

$$
E\left(\varphi(t), \dot{\varphi}(t) ; \Omega_{-}(t)\right) \leqq E(\varphi(0), \dot{\varphi}(0) ; \Omega)
$$

and

$$
E\left(\varphi(t), \dot{\varphi}(t) ; C \Omega_{+}(t)\right) \leqq E(\varphi(0), \dot{\varphi}(0) ; C \Omega),
$$

where $C$ denotes the complement in $\mathbb{R}^{n}$.

Proof. Without loss of generality, we can assume that $\Omega=B(0, L)$ and that $t$ is positive. We choose a function $m \in \mathscr{C}^{\infty}\left(\mathbb{R}, \mathbb{R}^{+}\right)$with $m(s)=0$ for $s \leqq 0, m(s)=1$ for $s \geqq 1$ and $0 \leqq m^{\prime}(s) \leqq 2$, and we define $m_{\varepsilon}(s)=m(s / \varepsilon)$. By a computation similar to those contained in the proofs of Proposition 3.6 and of Lemma 4.2 of [11] and an elementary argument of regularization in time, we obtain

$$
\begin{aligned}
& \int d x m_{\varepsilon}(L-t-|x|)\left\{\left|H_{j} \dot{\varphi}\right|^{2}+\left|H_{j} \nabla \varphi\right|^{2}+V\left(H_{j} \varphi\right)\right\}(t, x) \\
& \leqq \\
& \quad d x m_{\varepsilon}(L-|x|)\left\{\left|H_{j} \dot{\varphi}\right|^{2}+\left|H_{j} \nabla \varphi\right|^{2}+V\left(H_{j} \varphi\right)\right\}(0, x) \\
& \quad+\int_{0}^{t} d \tau \int d x m_{\varepsilon}(L-\tau-|x|) 2 \operatorname{Re} \overline{H_{j} \dot{\varphi}}\left(f\left(H_{j} \varphi\right)-H_{j} f(\varphi)\right)(\tau, x),
\end{aligned}
$$

where $H_{j}$ is the regularizing operator defined in the Appendix. We next let $j$ tend to infinity and $\varepsilon$ tend to zero in that order. The left-hand side and the first term in the right-hand side of $(4.28)$ tend to $E\left(\varphi(t), \dot{\varphi}(t) ; \Omega_{-}(t)\right)$ and $E(\varphi(0), \dot{\varphi}(0) ; \Omega)$ respectively, by the same argument as in the proof of Proposition 3.6. Note however that the assumption that $\varphi(t) \in L^{p+1}$ for some $t \in \mathbb{R}$ is not needed here, because the function $m_{\varepsilon}(L-\tau-|\cdot|)$ has compact support. We next prove that the second term in the righthand side of (4.28) tends to zero when $j \rightarrow \infty$ for fixed $\varepsilon$, by proving that the contributions of $f\left(H_{j} \varphi\right)$ and $H_{j} f(\varphi)$ both tend to their common formal limit. The argument is almost the same as in the proof of (3.49), (3.50) with an additional complication due to the function $\tilde{m}(\tau, x) \equiv m_{\varepsilon}(L-\tau-|x|)$. Instead of (3.53), we obtain the estimate

$$
\begin{aligned}
& \left|\int_{0}^{t} d \tau\left\langle H_{j} \dot{\varphi}(\tau), \tilde{m} f\left(H_{j} \varphi(\tau)\right)\right\rangle\right| \\
& \leqq C\left\|\dot{\varphi} ; L^{q}\left([0, t], \dot{B}_{r}^{\rho-1}\right)\right\|\left\|\tilde{m} f\left(H_{j} \varphi\right) ; L^{\bar{q}}\left([0, t], \dot{B}_{\bar{r}}^{1-\rho}\right)\right\| \\
& \leqq C t^{\zeta}\left\|\dot{\varphi} ; L^{q}\left([0, t], \dot{B}_{r}^{\rho-1}\right)\right\|\left\{\left\|\tilde{m} ; L^{\infty}\left([0, t], L^{\infty}\right)\right\|+\left\|\tilde{m} ; L^{\infty}\left([0, t], \dot{B}_{\ell}^{1-\rho}\right)\right\|\right\} \\
& \cdot\left\|\varphi ; L^{q}\left([0, t], \dot{B}_{r}^{\rho}\right)\right\|^{p},
\end{aligned}
$$

where $n / \ell=1-\rho \leqq 1 / 2$, the last inequality follows from a straightforward extension of Lemma 2.3 part (1), the first norm of $\tilde{m}$ is 1 and the second one is finite since 
$\tilde{m} \in \mathscr{C}^{\infty}$ and $\tilde{m}$ has compact support as a function of $x$. The same estimate holds for the contribution of $H_{j} f(\varphi)$ to (4.28). With that modification included, the proof proceeds as that of energy conservation in Proposition 3.6 through the use of the Lebesgue dominated convergence theorem in the time integral. QED

We now recall the basic decay estimate [21]. The result given in Lemma 4.3 below and its formal algebraic derivation are again the same as that for the NLKG equation given in Lemma 4.3 of [11], with a somewhat different proof. We define

$$
W_{1}(z) \equiv \bar{z} f(z)-V(z) .
$$

We also introduce the functions $g(x)=\left(1+|x|^{2}\right)^{-1 / 2}$ and $g_{1}=\nabla \cdot(x g)$. One checks easily that $(n-1) g \leqq g_{1} \leqq n g$ and that $\Delta g_{1} \leqq 0$ for $n \geqq 3$.

Lemma 4.3. Let $n \geqq 3$. Let $f$ satisfy (A1b) with p satisfying (3.30) and (A2). Let $\varphi$ be a finite energy solution of the equation (1.1). Then for any $s$ and $t$ in $\mathbb{R}, s \leqq t, \varphi$ satisfies the inequality

$$
\begin{aligned}
& \int_{s}^{t} d \tau \int d x g_{1}(x) W_{1}(\varphi(\tau, x)) \\
& \quad \leqq \operatorname{Re}\{\langle\dot{\varphi}(s),(x g \cdot \nabla+\nabla \cdot \dot{x g}) \varphi(s)\rangle-\langle\dot{\varphi}(t),(x g \cdot \nabla+\nabla \cdot x g) \varphi(t)\rangle\} .
\end{aligned}
$$

Proof. By the same algebraic computation as in the proof of Lemma 4.3 of [11] and an elementary argument of regularization in time, we obtain

$$
\begin{aligned}
\int_{s}^{t} d \tau & \int d x g_{1} W\left(H_{j} \varphi(\tau)\right) \\
= & \operatorname{Re}\left\{\left\langle H_{j} \dot{\varphi}(s),(x g \cdot \nabla+\nabla \cdot x g) H_{j} \varphi(s)\right\rangle-\left\langle H_{j} \dot{\varphi}(t),(x g \cdot \nabla+\nabla \cdot x g) H_{j} \varphi(t)\right\rangle\right\} \\
& \quad+\int_{s}^{t} d \tau \operatorname{Re}\left\langle H_{j} \varphi(\tau),[\Delta, x g] \cdot \nabla H_{j} \varphi(\tau)\right\rangle \\
& \quad+\int_{s}^{t} d \tau \operatorname{Re}\left\langle f\left(H_{j} \varphi(\tau)\right)-H_{j} f(\varphi(\tau)),(x g \cdot \nabla+\nabla \cdot x g) H_{j} \varphi(\tau)\right\rangle .
\end{aligned}
$$

where $H_{j}$ is the regularizing operator defined in the Appendix. The first integral in the right-hand side of (4.32) is negative (see the proof of Lemma 5.2 of [8]). We next let $j$ tend to infinity. By the same argument as in the proof of Proposition 3.6, the lefthand side of (4.32) converges to that of (4.31). Note in particular that

with

$$
\left\|g_{1} W_{1}(\varphi)\right\|_{1} \leqq C\left\|g_{1}\right\|_{\ell}\|\varphi\|_{2^{*}}^{p+1}
$$

$$
(p-1)(1 / 2-1 / n)=2 / n-1 / \ell,
$$

or equivalently $\ell=n / \zeta$ so that $\ell>n$ and $\left\|g_{1}\right\|_{\ell}<\infty$ for $\zeta<1$. In addition, the first two terms in the right-hand side of (4.32) converge to the right-hand side of (4.31). We next prove that the last integral in (4.32) tends to zero when $j \rightarrow \infty$ by proving that the contributions of $f\left(H_{j} \varphi\right)$ and of $H_{j} f(\varphi)$ both tend to their common formal limit. We use again the same argument as in the proof of (3.49), (3.50), now with an additional complication due to the function $x g$. In particular, instead of (3.53), we 
estimate

$$
\begin{aligned}
\mid \int_{s}^{t} d \tau & \left\langle f\left(H_{j} \varphi(\tau)\right),(x g \cdot \nabla+\nabla \cdot x g) H_{j} \varphi(\tau)\right\rangle \mid \\
\leqq & C \int_{s}^{t} d \tau\left\{\left\|f\left(H_{j} \varphi(\tau)\right) ; \dot{B}_{\dot{r}}^{1-\rho}\right\|\left\|\varphi(\tau) ; \dot{B}_{r}^{\rho}\right\|\|x g\|_{\infty}\right. \\
& +\left\|f\left(H_{j} \varphi(\tau)\right) ; \dot{B}_{\bar{r}}^{1-\rho-\varepsilon}\right\|\left\|\varphi(\tau) ; \dot{B}_{r}^{\rho}\right\|\left\|x g ; \dot{B}_{k}^{1-\rho}\right\| \\
& \left.+\left\|f\left(H_{j} \varphi(\tau)\right) ; \dot{B}_{\bar{r}}^{1-\rho}\right\|\left\|\varphi(\tau) ; \dot{B}_{r}^{\rho-\varepsilon}\right\|\left\|x g ; \dot{B}_{t}^{\rho}\right\|\right\}
\end{aligned}
$$

by a straightforward extension of Lemma 2.3 part (1), and with $\varepsilon>0, n / k=1-\rho-\varepsilon$, $n / \ell=\rho-\varepsilon$. Assuming for the moment that the last two norms of $x g$ are finite, and taking $\rho \geqq 1 / 2$, we estimate the last two norms of $f$ as

$$
\begin{aligned}
\left\|f\left(H_{j} \varphi(\tau)\right) ; \dot{B}_{\vec{r}}^{1-\rho}\right\| & \leqq C\left\|\varphi(\tau) ; \dot{B}_{r}^{\rho}\right\|^{p} \\
\left\|f\left(H_{j} \varphi(\tau)\right) ; \dot{B}_{\vec{r}}^{1-\rho-\varepsilon}\right\| & \leqq C\left\|\varphi ; \dot{B}_{r}^{\rho}\right\|^{p-1}\left\|\varphi ; \dot{B}_{r}^{\rho-\varepsilon}\right\|
\end{aligned}
$$

by Lemma $2.3 \operatorname{part}(1)$, with $(\rho+\delta(r)-1 \equiv) \sigma=(1-\zeta) /(p+1)$. We then estimate the time integrals in (4.34) by the Hölder inequality and (noting that $\|x g\|_{\infty}=1$ ) continue (4.34) as

$$
\begin{aligned}
\cdots \leqq & C\left\|\varphi ; L^{q}\left([s, t], \dot{B}_{r}^{\rho}\right)\right\|^{p+1}|t-s|^{\zeta} \\
& +C\left\|\varphi ; L^{q}\left([s, t], \dot{B}_{r}^{\rho}\right)\right\|^{p}\left\|\varphi ; L^{q^{\prime}}\left([s, t], \dot{B}_{r}^{\rho-\varepsilon}\right)\right\||t-s|^{\zeta+\varepsilon} \\
& \cdot\left\{\left\|x g ; \dot{B}_{k}^{1-\rho}\right\|+\left\|x g ; \dot{B}_{\ell}^{\rho}\right\|\right\}
\end{aligned}
$$

with $1 / q=\sigma$ and $1 / q^{\prime}=\sigma-\varepsilon$. The same estimate as (4.34) (4.35) holds for the contribution of $H_{j} f(\varphi)$ to the last term in (4.32). With those estimates available, the proof proceeds as that of energy conservation in Proposition 3.6 through the use of the dominated convergence theorem in the time integral.

It remains to be proved that the norms of $x g$ that occur in (4.35) are finite. We consider only the last one. By an elementary computation, we obtain, for any $x, x^{\prime} \in \mathbb{R}^{n}$,

$$
\left|x g(x)-x^{\prime} g\left(x^{\prime}\right)\right| \leqq \operatorname{Min}\left\{2,\left|x-x^{\prime}\right|\left(g(x)+g\left(x^{\prime}\right)\right)\right\} .
$$

Using the integral form of the Besov space norms (see (2.24)), we estimate

$$
\left\|x g ; \dot{B}_{\ell}^{\rho}\right\| \leqq C\left\{\int_{0}^{\infty} t^{-1} d t\left\{t^{-\rho} \operatorname{Sup}_{|y| \leqq t}\left\|x g-\tau_{y} x g\right\|_{\ell}\right\}^{2}\right\}^{1 / 2} .
$$

Using (4.36), we estimate the $L^{\ell}$ norm in two ways. For $|y| \leqq 1$, we obtain from the second term in the minimum in (4.36),

$$
\left\|x g-\tau_{y} x g\right\|_{\ell} \leqq 2|y|\|g\|_{\ell} .
$$

For $|y| \geqq 1$, we use the first term in the minimum for $|x+y / 2| \leqq 2|y|$ and the second term for $|x+y / 2| \geqq 2|y|$ and obtain

$$
\left\|x g-\tau_{y} x g\right\|_{\ell} \leqq C|y|^{n / \ell} .
$$


Substituting the estimates (4.38) for $|y| \leqq 1$ and (4.39) for $|y| \geqq 1$ into (4.37) and using the fact that $n / \ell=\rho-\varepsilon$ shows that $x g \in \dot{B}_{\ell}^{\rho}$. QED

In the same way as in [8] and [11], the estimate of Lemma 4.3 will be used through its following consequence.

Lemma 4.4. Let $n \geqq 3$. Let $f$ and $\varphi$ be as in Lemma 4.3 and assume in addition that $W_{1} \geqq 0$. Then for any $\varepsilon>0, a_{0}>0$ and $\ell_{0}>0$, there exists $b_{0}>0$, depending only on $\varepsilon, a_{0}, \ell_{0}$ and on the energy $E$ of $\varphi$, and there exists $c$ such that $a_{0} \leqq c \leqq b_{0}-\ell_{0}$ and

$$
\int_{c}^{c+\ell_{0}} d \tau \int_{|x| \leqq 2 \tau} d x W_{1}(\varphi(\tau, x)) \leqq \varepsilon .
$$

One can take

$$
b_{0}=\left(a_{0}+\ell_{0}+1\right) \exp \left\{4 E \ell_{0} \varepsilon^{-1}(n-1)^{-1}\right\} .
$$

Proof. The proof is the same as that of Lemma 4.4 of [11].

The next step in the argument consists in proving that an arbitrary finite energy solution of the equation (1.1) is arbitrarily small in arbitrarily large intervals of time provided those intervals are located sufficiently far. That result requires a repulsivity property of the interaction term $f$, which we state as follows

(A3) There exists $C>0$ and $p_{4}, p_{5}$ with $1 \leqq p_{4} \leqq p_{5}<\infty$ such that for all $\rho \in \mathbb{R}^{+}$,

$$
W_{1}(\rho) \geqq C \operatorname{Min}\left(\rho^{p_{4}+1}, \rho^{p_{5}+1}\right) \text {. }
$$

We can now prove the following result, in close analogy with Lemma 4.5 of [11].

Lemma 4.5. Let $n \geqq 4$, let $f$ satisfy (A1b) with p satisfying (4.13), (A2) with $V \geqq 0$ and (A3). Let $r, \rho, \sigma$ and $q$ satisfy (2.5) and $0<\sigma=1 / q$. Let $\varphi$ be a finite energy solution of the equation (1.1). Then for any $\varepsilon>0$ and any $\ell>0$, there exists $a>0$ such that

$$
\left\|\varphi ; \ell^{\infty}\left(L^{q},[a, a+\ell], \dot{B}_{r}^{\rho}\right)\right\| \leqq \varepsilon .
$$

Proof. The proof is the same as that of Lemma 4.5 of [11] with only one difference. Since now the energy does not contain the $L^{2}$ norm, the contribution of the region $t-\theta_{2} \leqq \tau \leqq t-\theta_{1},|x|>2 \tau$ has to be estimated by using the norm of $\varphi$ in $L^{2^{*}}$, namely by taking $v_{3}=2^{*}$. The local energy estimate of Lemma 4.2 has then to be supplemented by a decay estimate for the norm of $\varphi$ in $L^{2^{*}}$ replacing (4.38) of [11]. That estimate is given in Lemma 4.6 below. With that estimate available, the proof proceeds as that of Lemma 4.5 of [11]. QED

Lemma 4.6. Let $n \geqq 3$. Let $f$ satisfy (A1b) with $p$ satisfying (3.30) and (A2) with $V \geqq 0$. Let $\varphi$ be a finite energy solution of the equation (1.1). Then

$$
\lim _{t \rightarrow \infty}\left\|\varphi(t) ; L^{2 *}(C B(0,2 t))\right\|=0 .
$$

Proof. Let $1<a<2$, let $\chi \in \mathscr{C}^{\infty}\left(\mathbb{R}^{n}\right)$ satisfy $\chi(x)=0$ for $|x| \leqq a, \chi(x)=1$ for $|x| \geqq 2$ and $0 \leqq \chi \leqq 1$, and let $\chi_{t}(x)=\chi(x / t)$. Then for all $t \geqq 0$,

$$
\begin{aligned}
\left\|\varphi(t) ; L^{2^{*}}(G B(0,2 t))\right\| \leqq & \left\|\chi_{t} \varphi(t)\right\|_{2^{*}} \leqq\left\|\left(\nabla \chi_{t}\right) \varphi(t)\right\|_{2}+\left\|\chi_{t} \nabla \varphi(t)\right\|_{2} \\
\leqq & t^{-1}\|\nabla \chi\|_{\infty}\left\|\varphi(t) ; L^{2}(B(0,2 t) \backslash B(0, a t))\right\| \\
& \left.+\|\chi\|_{\infty} \| \nabla \varphi(t) ; C B(0, a t)\right) \| .
\end{aligned}
$$


The first norm of $\varphi$ in the last member of (4.45) is estimated by writing $\varphi$ as the integral of $\dot{\varphi}$ and estimating the local $L^{2}$ norm of the latter by Lemma 4.2, while the local $L^{2}$ norm of $\nabla \varphi$ is estimated directly by the same lemma. We can then continue (4.45) as

$$
\begin{aligned}
\cdots \leqq & \|\nabla \chi\|_{\infty}\left\{t^{-1}\left\|\varphi(0) ; L^{2}(B(0,2 t) \backslash B(0, a t))\right\|\right. \\
& \left.+E(0, B(0,3 t) \backslash B(0,(a-1) t))^{1 / 2}\right\}+E(0, \zeta B(0,(a-1) t))^{1 / 2}
\end{aligned}
$$

The remaining norm of $\varphi(0)$ is estimated by the Hölder inequality, so that (4.46) can be continued as

$$
\begin{aligned}
& \cdots \leqq\|\nabla \chi\|_{\infty} 2 \omega_{n}^{1 / n}\left\|\varphi(0) ; L^{2^{*}}(B(0,2 t) \backslash B(0, a t))\right\| \\
& +\left(1+\|\nabla \chi\|_{\infty}\right) E(0, C B(0,(a-1) t))^{1 / 2} \text {, }
\end{aligned}
$$

where $\omega_{n}$ is the volume of the unit ball in $\mathbb{R}^{n}$. The result follows from (4.47), from the fact that $\varphi(0) \in L^{2 *}$ and from Lemma 4.2. QED

In order to proceed further, we need to prove that some norm of an arbitrary finite energy solution of the equation (1.1) satisfies a superlinear integral inequality in addition to the sublinear inequality used previously (see (4.25)). For that purpose, we need the following estimate.

Lemma 4.7. Let $n \geqq 4$. Let $f$ satisfy (A1a) for two values $p_{1}$ and $p_{2}$ with

$$
p_{2}-1<4 /(n-2)<p_{1}-1 \text {. }
$$

Let $r$ and $\sigma$ satisfy $\gamma(r)>1, \gamma(r)$ sufficiently close to 1 , and $\sigma<1 / 2, \sigma$ sufficiently close to $1 / 2$. Then there exists $\gamma_{1}$ and $\gamma_{2}$ satisfying $0 \leqq \gamma_{2}<1<\gamma_{1}$ and there exists $v$ satisfying $v>1, v \sigma \leqq 1$ and $(v-1) \sigma<1-\gamma_{2}$, such that for all $\varphi \in \dot{B}_{r}^{\rho} \cap \dot{H}_{2}^{1}$ and all $t>0$, the following estimate holds

$$
\left\|K(t) f(\varphi) ; \dot{B}_{r}^{\rho}\right\| \leqq \operatorname{Min}_{i=1,2}\left(|t|^{-\gamma_{i}}\right) M\left(\left\|\varphi ; \dot{H}_{2}^{1}\right\|\right)\left\|\varphi ; \dot{B}_{r}^{\rho}\right\|^{\nu} .
$$

Proof. Without loss of generality, we assume that $\left(p_{1}-1\right)(n / 2-1)=2(1+\varepsilon)$ and $\left(p_{2}-1\right)(n / 2-1)=2-\varepsilon$ and we take $\gamma(r)=1+\varepsilon$ with $\varepsilon>0, \varepsilon$ suitably small. We obtain the two estimates of (4.49) by applying successively (2.3) (see also (3.5), (3.6)) and Lemma 4.1 with $\eta=0$ and $\lambda=\sigma+\alpha(\ell)$, once with $p=p_{1}, \gamma_{1}=\gamma\left(\ell_{1}\right)=1+\varepsilon$, and once with $p=p_{2}, \gamma_{2}=\gamma\left(\ell_{2}\right)=1-2 \varepsilon$ and with $(v-1) \sigma=\varepsilon$, which fulfills the relation (4.3) or equivalently

$$
(p-1)(n / 2-1)=1+\gamma(\ell)+(v-1) \sigma
$$

in both cases. Furthermore $(v-1) \sigma=\varepsilon<1-\gamma_{2}=2 \varepsilon$ and $v \sigma=\sigma+\varepsilon \leqq 1$. The upper interpolation condition (4.2) is seen to be satisfied in both cases for $\varepsilon$ sufficiently small and $\sigma$ sufficiently close to $1 / 2$ by an explicit computation for which we refer to the proof of Lemma 3.3 in [9]. QED

It follows immediately from Lemma 4.7 that under suitable assumptions on $f$ and on $\rho, r, q$, any finite energy solution of the equation (1.1) satisfies the following properties. The norm $k(t)$ defined by (4.24) belongs to $\ell^{\infty}\left(L^{q}\right)$ by Proposition 4.1, is small in large intervals by Lemma 4.5 and satisfies a superlinear inequality

$$
k \leqq k_{0}+\mu * k^{\nu}
$$


where $v>1$ and

$$
\begin{array}{lll}
\mu(t)=\operatorname{Min}_{i=1,2}|t|^{-\gamma_{i}} & \text { for } & t \geqq 0 \\
\mu(t)=0 & \text { for } & t \leqq 0,
\end{array}
$$

by Lemma 4.7. From that information, the dispersive properties of such solutions follow by the same abstract arguments as in the case of the NLS equation [8]. We now state the final result.

Proposition 4.2. Let $n \geqq 4$. Let $f$ satisfy (A1b) for two values $p_{1}$ and $p_{2}$ satisfying (4.48), (A2) with $V \geqq 0$ and (A3). Then

(1) Any finite energy solution of the equation (1.1) belongs to $\mathscr{Y}(\mathbb{R})$.

(2) The wave operators $\Omega_{ \pm}$defined in Proposition 3.7 part (2) are surjective in $X_{e}$.

Indication of Proof. It follows from Proposition 4.1, Lemmas 4.5, the integral inequality (4.51) and from Lemmas 5.10 and 5.11 of [8] that any finite energy solution $\varphi$ of the equation (1.1) belongs to $L^{q}\left(\mathbb{R}, \dot{B}_{r}^{\rho}\right)$ for the values of $\rho, r, q$ that appear in Lemma 4.7. By interpolation with the boundedness of $\varphi$ in $\dot{H}_{2}^{1}$ and the Sobolev inequalities, this implies that $\varphi \in L^{q}\left(\dot{B}_{r}^{\rho}\right)$ for all $\rho, r, \sigma$ and $q$ satisfying (2.5) and $1 / q=\sigma$, except for the boundary values corresponding to $\gamma(r)=2 \sigma$. Finally, the whole region (2.5) is recovered by applying Proposition 2.3. QED

In the framework of Scattering theory, Proposition 4.2 part (2) means that the wave operators obtained in Proposition 3.7 are asymptotically complete in $X_{e}$.

\section{Appendix}

In this appendix, we collect the definition and some basic properties of the homogeneous Besov and Sobolev space of arbitrary order (see [1] for general information on those spaces). We discuss in particular the problem of the choice of representatives (see [3] for that question). We also discuss the meaning attached to the equation (1.1) and its equivalence with the integral equation (2.33).

We denote by $\mathscr{F}$ the Fourier transform and we write $\hat{v}=\mathscr{F} v$ for any $v \in \mathscr{S}^{\prime} \equiv \mathscr{S}^{\prime}\left(\mathbb{R}^{n}\right)$. We define the subspaces $\mathscr{Z} \equiv \mathscr{Z}\left(\mathbb{R}^{n}\right)$ and $\mathscr{D}{ }_{0}$ of $\mathscr{S} \equiv \mathscr{S}\left(\mathbb{R}^{n}\right)$ by

$$
\mathscr{Z}=\left\{u \in \mathscr{S}: D^{\alpha} \hat{u}(0)=0 \text { for any multiindex } \alpha\right\}
$$

and

$$
\hat{\mathscr{D}}_{0}=\left\{u \in \mathscr{S}: \hat{u} \in \mathscr{C}_{0}^{\infty}\left(\mathbb{R}^{n} \backslash\{0\}\right)\right\} .
$$

Clearly $\mathscr{Z}$ is a closed subspace of $\mathscr{S}$, so that $\mathscr{Z}$ is reflexive, while $\mathscr{D}_{0}$ is easily seen to be dense in $\mathscr{Z}$. The dual of the inclusion map $\mathscr{Z} \subset \mathscr{S}$ is a surjection $\pi$ from $\mathscr{S}^{\prime}$ to $\mathscr{Z}^{\prime}$, the kernel of which consists of the polynomials $\mathscr{P}$, so that $\mathscr{Z}^{\prime}=\mathscr{S}^{\prime} / \mathscr{P}$.

Let $\hat{\psi}_{0} \in \mathscr{C}_{0}^{\infty}\left(\mathbb{R}^{n}\right)$ with $0 \leqq \hat{\psi}_{0} \leqq 1, \hat{\psi}_{0}(\xi)=1$ for $|\xi| \leqq 1$ and $\hat{\psi}_{0}(\xi)=0$ for $|\xi| \geqq 2$. For any $j \in \mathbb{Z}$ define $\hat{\psi}_{j}(\xi)=\hat{\psi}_{0}\left(2^{-j} \xi\right)$ and $\hat{\varphi}=\hat{\psi}_{j}-\hat{\psi}_{j-1}$ so that

$$
\operatorname{Supp} \hat{\varphi}_{j} \subset\left\{\xi: 2^{j-1} \leqq|\xi| \leqq 2^{j+1}\right\}
$$

and, for any $\xi \neq 0, \hat{\psi}_{j}(\xi)=\sum_{\ell \leqq j} \hat{\varphi}_{\ell}(\xi)$ with at most two non-vanishing terms in the sum. 
By homogeneity $\left\|\psi_{j}\right\|_{1}=\left\|\psi_{0}\right\|_{1}$ and $\left\|\varphi_{j}\right\|_{1}=\left\|\varphi_{0}\right\|_{1}$ for any $j \in \mathbb{Z}$.

With any $u \in \mathscr{S}^{\prime}$ we can associate the sequence of functions $\left\{\varphi_{j} * u \equiv u_{j}\right\}, j \in \mathbb{Z}$. That sequence is actually defined for $u \in \mathscr{Z}^{\prime}$ since $u_{j}=0$ for $u \in \mathscr{P}$. For any $\rho \in \mathbb{R}$ and any $r$ and $s$ with $1 \leqq r, s \leqq \infty$ we define the homogeneous Besov spaces

$$
\dot{B}_{r s}^{\rho}=\left\{u \in \mathscr{Z}^{\prime}:\left\{\sum_{j} 2^{j \rho s}\left\|\varphi_{j} * u\right\|_{r}^{s}\right\}^{1 / s} \equiv\left\|u ; \dot{B}_{r s}^{\rho}\right\|<\infty\right\},
$$

and the auxiliary spaces

$$
\dot{F}_{r s}^{\rho}\left\{u \in \mathscr{Z}^{\prime}:\left\|\left\{\sum_{j} 2^{j \rho s}\left|\varphi_{j} * u\right|^{s}\right\}^{1 / s}\right\|_{r} \equiv\left\|u ; \dot{F}_{r s}^{\rho}\right\|<\infty\right\}
$$

with obvious modifications if $s=\infty$. The factor $2^{\rho}$ in the sums mimicks a derivative of order $\rho$. Equipped with the norm given by (A.3) and (A.4) the spaces $\dot{B}_{r s}^{\rho}$ and $\dot{F}_{r s}^{\rho}$ are Banach spaces. They satisfy $\dot{F}_{r s}^{\rho} \subset \dot{B}_{r s}^{\rho}$ if $1 \leqq r \leqq s \leqq \infty$ and $\dot{B}_{r s}^{\rho} \subset \dot{F}_{r s}^{\rho}$ if $1 \leqq s \leqq r \leqq \infty$.

We next define the homogeneous Sobolev spaces. For any $\rho \in \mathbb{R}$, we define the operator $\omega^{\rho}$ by $\hat{\omega}^{\rho} \dot{u}(\xi)=|\xi|^{\rho} \hat{u}(\xi)$ for any $u \in \mathscr{Z}$. Then $\omega^{\rho}$ is an homeomorphism of $\mathscr{Z}$ onto itself, and therefore induces an homeomorphism of $\mathscr{Z}^{\prime}$ onto itself, still denoted by $\omega^{\rho}$. For any $\rho$, for any $r$ with $1 \leqq r<\infty$, we define the homogeneous Sobolev spaces $\dot{H}_{r}^{\rho}$ by

$$
\dot{H}_{r}^{\rho}=\omega^{-\rho} \pi\left(L^{r}\right)
$$

Any $u \in \pi\left(L^{r}\right)$ is the image of only one representative $u_{R} \in L^{r}$. Equipped with the norm $\left\|u ; \dot{H}_{r}^{\rho}\right\| \equiv\left\|\left(\omega^{\rho} u\right)_{R}\right\|_{r}, \dot{H}_{r}^{\rho}$ is a Banach space. The homogeneous Besov and Sobolev spaces can be compared by using the (non-trivial) fact that $\dot{F}_{r 2}^{\rho}=\dot{H}_{r}^{\rho}$ for $1<r<\infty$ so that the previous embeddings imply $\dot{B}_{r 2}^{\rho} \subset \dot{H}_{r}^{\rho} \subset \dot{B}_{r r}^{\rho}$ if $2 \leqq r<\infty$ and $\dot{B}_{r r}^{\rho} \subset \dot{H}_{r}^{\rho} \subset \dot{B}_{r 2}^{\rho}$ if $1<r \leqq 2$. The Sobolev embeddings for the Besov spaces take the form

$$
\dot{B}_{r s}^{\rho} \subset \dot{B}_{r^{\prime} s}^{\rho^{\prime}}
$$

with $n / r-\rho=n / r^{\prime}-\rho^{\prime}, 1 \leqq r \leqq r^{\prime} \leqq \infty$ and $1 \leqq s \leqq \infty$.

Another embedding of interest is contained in the following lemma.

Lemma A.1. Let $1 \leqq \ell, m, m^{\prime} \leqq \infty, m^{\prime} \leqq m, \lambda \in \mathbb{R}$ and $\varepsilon>0$. Then $\dot{B}_{\ell, m^{\prime}}^{\lambda} \supset \bigcap_{ \pm} \dot{B}_{\ell, m}^{\lambda \pm \varepsilon}$, and the following estimate holds

$$
\left\|u ; \dot{B}_{\ell, m}^{\lambda}\right\| \leqq C\left\|u ; \dot{B}_{\ell, m^{\prime}}^{\lambda}\right\| \leqq C \prod_{ \pm}\left\|u ; \dot{B}_{\ell, m}^{\lambda \pm \varepsilon}\right\|^{1 / 2} .
$$

Proof. The result follows from the definition of Besov spaces through a dyadic decomposition by a simple use of the Hölder inequality. QED

For a number of arguments we need to approximate elements of $\mathscr{Z}^{\prime}$ by smooth functions. For any $j, k \in \mathbb{Z}^{+}$we define the regularizing operators

$$
H_{j k} u=\left(\psi_{j}-\psi_{-k}\right) * u
$$

for any $u \in \mathscr{S}^{\prime}$ and $H_{j} \equiv H_{j j}$. Clearly $H_{j k} u \in \mathscr{C}^{\infty}$ with at most polynomial increase. The operators $H_{j k}$ are bounded uniformly with respect to $j, k$ and converge strongly when $j$ and (or) $k \rightarrow \infty$ in a number of spaces. By the definition (A.1) the operators $H_{j k}$ are uniformly bounded with respect to $j$ and $k$ in all $\dot{B}_{r s}^{\rho}$ and converge strongly to 1 when 
$j, k \rightarrow \infty$ for $s<\infty$. The operators $H_{j k}$ converge strongly to 1 in $\hat{\mathscr{D}}_{0}$ since $H_{j k} u$ is independent of $j, k$ for $u \in \hat{\mathscr{D}}_{0}$ and $j, k$ sufficiently large. In addition $H_{j k}$ converges to $H_{\infty k}$ when $j \rightarrow \infty$ strongly in $\mathscr{S}$ and therefore in $\mathscr{S}^{\prime}$, and $H_{j k}$ converges to $\mathbb{1}$ when $j, k \rightarrow \infty$ strongly in $\mathscr{Z}$ and therefore in $\mathscr{Z}^{\prime}$. Furthermore the operators $H_{j k}$ are uniformly bounded with respect to $j$ and $k$ in $L^{r}$ since

$$
\left\|H_{j k} u\right\|_{r} \leqq\left\|\psi_{j}-\psi_{-k}\right\|_{1}\|u\|_{r} \leqq 2\left\|\psi_{0}\right\|_{1}\|u\|_{r} .
$$

Since $\hat{\mathscr{D}}_{0}$ is dense in $L^{r}$ for $1<r<\infty, H_{j k}$ converges strongly to $\mathbb{1}$ in $L^{r}$ when $j, k \rightarrow \infty$.

The locally regularized functions $H_{j k} u$, with $u \in \mathscr{S}^{\prime}$, in general do not decay at infinity. In some applications it will be useful to introduce an additional cut off at infinity in space. For that purpose we choose a function $g_{0}$ with $\hat{g}_{0} \in \mathscr{C}_{0}^{\infty}, \hat{g}_{0} \geqq 0$, Supp $\hat{g}_{0} \subset\{\xi:|\xi|<1 / 2\},\left\|\hat{g}_{0}\right\|_{1}=1$ and, for any $j \in \mathbb{Z}$, we define $\hat{g}_{j}(\xi)=2^{n j} \hat{g}_{0}\left(2^{j} \xi\right)$. The operators of multiplication by $g_{j}$ are uniformly bounded in norm with respect to $j$ in all the $L^{r}, 1 \leqq r \leqq \infty$, and converge to 1 strongly in $L^{r}$ for $1 \leqq r<\infty$ when $j \rightarrow \infty$. We define the operators $R_{j}$ by $R_{j} u=g_{j} H_{j j} u$ for any $u \in \mathscr{S}^{\prime}$, so that $R_{j} u \in \hat{\mathscr{D}}_{0}$. Note also that

$$
\text { Supp } R_{j} u \subset\left\{\xi: 2^{-(j+1)} \leqq|\xi| \leqq 2^{j+1}+2^{-(j+1)}\right\} .
$$

Clearly the operators $R_{j}$ are uniformly bounded in norm with respect to $j$ in $L^{r}$, $1 \leqq r \leqq \infty$, and converge to 1 strongly in $L^{r}, 1<r<\infty$ when $j \rightarrow \infty$. We next study the behaviour of $R_{j}$ in the Besov spaces.

Lemma A.2. (1) The operators $R_{j}$ are uniformly bounded with respect to $j$ in $\dot{B}_{r s}^{\rho}$, with $\rho \in \mathbb{R}, 1 \leqq r \leqq \infty, 1 \leqq s \leqq \infty$.

(2) The operators $R_{j}$ converge to 1 when $j \rightarrow \infty$ strongly in $\dot{B}_{r s}^{\rho}$ with $\rho \in \mathbb{R}, 1 \leqq r<\infty$, $1 \leqq s<\infty$.

Proof. We prove only Part (2) which is slightly more complicated than Part (1). By

$$
R_{j} u-u=\left(g_{j}-1\right) H_{j} u+\left(H_{j} u-u\right),
$$

and by the convergence properties of $H_{j}$, it is sufficient to prove that $\left(g_{j}-1\right) H_{j} u \rightarrow 0$ in $\dot{B}_{r s}^{\rho}$. For that purpose we need an estimate in $\ell^{s}$ of the sequence $v=\left\{v_{m}\right\}$ defined by

$$
v_{m} \equiv 2^{\rho m}\left\|\varphi_{m} *\left(\left(g_{j}-1\right) H_{j} u\right)\right\|_{r} \leqq \sum_{-j<k \leqq j} 2^{\rho m}\left\|\varphi_{m} *\left(\left(g_{j}-1\right)\left(\varphi_{k} * u\right)\right)\right\|_{r} .
$$

By the support properties of $\hat{\varphi}_{k}$ and $\hat{g}_{j}$ the norms in the right-hand side of (A.8) are different from zero only for $|k-m| \leqq 2$. Using the Young inequality and the previous restriction on $k$ we obtain

$$
\left\|v ; \ell^{s}\right\| \leqq\left(\sum_{|\ell| \leqq 2} 2^{\rho \ell}\right)\left\|w ; \ell^{s}\right\| \leqq 5.4^{|\rho|}\left\|w ; \ell^{s}\right\|,
$$

where $w=\left\{w_{k}\right\}$ is the sequence

$$
w_{k}=2^{\rho k}\left\|\left(g_{j}-1\right)\left(\varphi_{k} * u\right)\right\|_{r}\left\|\varphi_{0}\right\|_{1}
$$

Since $u \in \dot{B}_{r s}^{\rho}$ the sequence $w$ is bounded uniformly in $j$ by a sequence in $\ell^{s}$ and each term tends to zero when $j \rightarrow \infty$, so that $w \rightarrow 0$ in $\ell^{s}$ when $j \rightarrow \infty$. QED

The elements of homogeneous Besov spaces are equivalence classes of distri- 
butions modulo polynomials. However we are interested in solving the equation (1.1) in a genuine distributional sense. Therefore we repeatedly face the problem of choosing distributional representatives of elements of $\dot{B}_{r s}^{\rho}$ spaces, and making sure that those choices are consistent with the various operations to be performed. This is all the more necessary as some of the statements and estimates, such as those of Lemma 2.3, explicitly require the use of representatives.

The problem of the choice of representatives has been studied in [3], from which we extract Lemma A.3 below. In order to state it, we define the space $\mathscr{S}_{0}^{\prime}$ of tempered distributions tending to zero at infinity as

$$
\mathscr{S}_{0}^{\prime}=\left\{u \in \mathscr{S}^{\prime}: d_{\lambda} u \rightarrow 0 \text { in } \mathscr{S}^{\prime} \text { when } \lambda \rightarrow 0\right\},
$$

where $d_{\lambda}$ is the dilation operator defined by $\left(d_{\lambda} u\right)(x)=u(x / \lambda)$.

Lemma A.3. Let $1 \leqq r, s \leqq \infty, n / \ell \equiv n / r-\rho>0$. Then

(1) Any $u \in \dot{B}_{r s}^{\rho}$ has a unique representative $u_{R}$ in $\mathscr{S}_{0}^{\prime}$. The map $u \rightarrow u_{R}$ is continuous from $\dot{B}_{r s}^{\rho}$ to $\mathscr{S}_{0}^{\prime}$ and $u_{R}$ is the limit of $H_{j} u$ in $\mathscr{S}^{\prime}$ when $j \rightarrow \infty$.

In addition

(2) If $\rho>0$, then $u_{R} \in L^{\ell+\varepsilon}+L^{\ell-\varepsilon}$ (for some $\varepsilon>0$ ). The map $u \rightarrow u_{R}$ is continuous from $\dot{B}_{r s}^{\rho}$ to $L^{\ell+\varepsilon}+L^{\ell-\varepsilon}$ and $u_{R}$ is the limit of $H_{j} u$ and of $R_{j} u$ in $L^{\ell+\varepsilon}+L^{\ell-\varepsilon}$ when $j \rightarrow \infty$.

(3) If $\rho \geqq 0, s \leqq 2$ and $\ell \geqq 2$, then $u_{R} \in L^{\ell}$. The map $u \rightarrow u_{R}$ is continuous from $\dot{B}_{r s}^{\rho}$ to $L^{\ell}$ and $u_{R}$ is the limit of $H_{j} u$ and of $R_{j} u$ in $L^{\ell}$ when $j \rightarrow \infty$.

Proof. We refer to [3] for Parts (1) and (2). Part (3) follows from the embedding (A.5) and the properties of $H_{j}$ and $R_{j}$. QED

In all the applications made in this paper the assumptions of Lemma A.3 Part (1) are satisfied. Whenever a choice of a representative is needed we select the representative described in that lemma, which we sometimes call the canonical representative. In order to simplify the notation we use the same symbol for the Besov space element and for its canonical representative. That occurs in particular in the following three cases:

(1) In the definition (2.1) of the energy space $X_{e}$ it is understood that the first component is the canonical representative (which is in $L^{2 *}$ ) of an element of $\dot{H}_{2}^{1}=\dot{B}_{2}^{1}$, according to Lemma A.3 part(3).

(2) The interaction term $f$ in (1.1), generally satisfying the assumption (A1a), is always to be applied in the ordinary sense to the canonical representative of its argument. Subsequent estimates such as the estimates (2.17), (2.19) and (2.21) of $f(\varphi)$ in Lemma 2.3 are then performed on the class of $f(\varphi)$ in a suitable Besov space. Clearly the functions $g_{1}$ and $g_{2}$ in the same estimates are also applied to the canonical representative of $\varphi$. Finally, by Lemma A.3 Part (2) and since $f(0)=0$, $f(\varphi)$ is the canonical representative of its class.

(3) The operators $K, \dot{K}, \ddot{K}, \ldots$ obviously commute with the regularisation operator $H_{j}$. It follows therefore from Lemma A.3 that they preserve the choice of the canonical representative whenever it exists. This remark applies in particular to the transition from $\Phi$ to $\widetilde{\Phi}$ defined by (2.38).

We now discuss the meaning of the integral equation (2.33) and its relation with the 
differential equation (1.1). We shall always work in situations where $f(\varphi) \in L_{\text {loc }}^{m}\left(I, \dot{B}_{\ell}^{\lambda}\right)$ for some interval $I$ of $\mathscr{R}$ with $\ell, m \geqq 1, \lambda>0, n / \ell-\lambda>0$. The integral which occurs in (2.35) is defined as a weak integral in $\mathscr{Z}^{\prime}$ by

$$
\left\langle\zeta, \int_{t_{0}}^{t} d \tau K(t-\tau) f(\varphi(\tau))\right\rangle \equiv \int_{t_{0}}^{t} d \tau\langle K(t-\tau) \zeta, f(\varphi(\tau))\rangle
$$

for all $t_{0}, t \in I$ and for all $\zeta \in \mathscr{Z}$. With the choice of the canonical representative of $\varphi$ and therefore of $f(\varphi)$, the integral is also defined weakly in $\mathscr{S}^{\prime}$. It follows from the available estimates and from Lemma A.3 and the subsequent discussion that the weak $\mathscr{S}^{\prime}$ integral is the canonical representative of the weak $\mathscr{Z}^{\prime}$ integral.

We next define the weak time derivative in $\mathscr{Z}^{\prime}$ (a similar definition holds in $\mathscr{S}^{\prime}$ ). Let $I$ be an open interval of $\mathbb{R}$ and let $\varphi \in L_{\text {loc }}^{q}(I, X), \dot{\varphi} \in L_{\text {loc }}^{q}(I, Y), q \geqq 1$, where $X$ and $Y$ are two Banach spaces continuously embedded in $\mathscr{Z}^{\prime}$ (or $\mathscr{S}^{\prime}$ ). We say that $\dot{\varphi}$ is the time derivative of $\varphi$ if the following equality holds in $\mathscr{D}^{\prime}(I)$

$$
d\langle\zeta, \varphi\rangle / d t=\langle\zeta, \dot{\varphi}\rangle
$$

for all $\zeta \in \mathscr{Z}$ (or $\zeta \in \mathscr{S}$ ).

The differential equation (1.1) and the integral equation (2.33) are equivalent in the following sense.

Proposition A.1. Let $I$ be an open interval of $\mathbb{R}$ and let $\varphi \in L^{q}\left(I, \dot{B}_{r}^{\rho}\right)$ be such that $f(\varphi) \in L^{m}\left(I, \dot{B}_{\ell}^{\lambda}\right)$, with $r, \ell, q, m \geqq 1$.

(1) Let $\varphi$ satisfy the equation (1.1) in the previous weak sense. Then $\varphi \in \mathscr{C}^{1}\left(I^{c}, X\right), \dot{\varphi} \in \mathscr{C}\left(I^{c}, X\right)$ where $I^{c}$ is the closure of $I$ in $\mathbb{R}, X=\dot{B}_{\ell}^{\lambda}+\dot{B}_{r}^{\rho-2}$ and, for all $s, t \in I^{c}, \varphi$ satisfies the equation

with

$$
\varphi(t)=A\left(s, \varphi^{(0)} ; \varphi\right)(t)
$$

$$
\varphi^{(0)}(t)=\dot{K}(t-s) \varphi(s)+K(t-s) \dot{\varphi}(s) .
$$

(2) Conversely let $\varphi^{(0)} \in L^{q}\left(I, \dot{B}_{r}^{\rho}\right)$ (this is not essential) be solution of the equation $\square \varphi^{(0)}=0$ and let $\varphi$ satisfy the equation (A.11) for some $s \in I^{c}$. Then $\varphi$ satisfies the equation (1.1) in the previous weak sense.

The proof of Proposition A.1 proceeds by a direct computation and duality arguments.

Acknowledgements. We are grateful to G. Bourdaud for enlightening conversations.

\section{References}

1. Bergh, J., Löfström, J.: Interpolation spaces. Berlin, Heidelberg, New York: Springer 1976

2. Birman, M. S., Solomjak, M. Z.: Vest, LSU 24 (1969). Translation: Vest. LSU Math. 2, 9-27 (1975)

3. Bourdaud, G.: Réalisation des espaces de Besov homogènes, Orsay preprint, 1986

4. Brenner, P.: Math. Z. 145, 251-254 (1975)

5. Brenner, P.: Math. Z. 186, 383-391 (1984)

6. Brenner, P.: J. Diff. Eq. 56, 310-344 (1985)

7. Ginibre, J., Velo, G.: J. Funct. Anal. 32, 33-71 (1979)

8. Ginibre, J., Velo, G.: J. Math. Pures Appl. 64, 363-401 (1985) 
9. Ginibre, J., Velo, G.: Math. Z. 189, 487-505 (1985)

10. Ginibre, J., Velo, G.: Ann. IHP (Anal. Non-lin.) 6, 15-35 (1989)

11. Ginibre, J., Velo, G.: Ann. IHP (Phys. Théor.) 43, 399-442 (1985)

12. Ginibre, J., Velo, G.: Ann. IHP (Phys. Théor.) 47, 221-261 (1987)

13. Ginibre, J., Velo, G.: Ann. IHP (Phys. Théor.) 47, 263-276 (1987)

14. Ginibre, J., Velo, G.: unpublished

15. Glassey, R. T., Pecher, H.: Manuscripta Math. 38, 387-400 (1982)

16. Hörmander, L.: The analysis of partial differential operators I. Berlin, Heidelberg, New York: Springer 1983

17. Kato, T.: Ann. IHP (Phys. Théor.) 46, 113-129 (1987)

18. Lin, J. E., Strauss, W.: J. Funct. Anal. 30, 245-263 (1978)

19. Mochizuki, K., Motai, M.: J. Math. Kyoto Univ. 25, 703-715 (1985)

20. Mochizuki, K., Motai, T.: The scattering theory for the non-linear wave equation with small data, preprint (1986)

21. Morawetz, C., Strauss, W.: Commun. Pure Appl. Math. 25, 1-31 (1972)

22. Pecher, H.: Math. Z. 150, 159-183 (1976)

23. Pecher, H.: Math. Z. 185, 261-270 (1984)

24. Strauss, W.: J. Funct. Anal. 2, 409-457 (1968)

25. Yajima, K.: Commun. Math. Phys. 110, 415-426 (1987)

Communicated by A. Jaffe

Received September 13, 1988 
